\title{
$U(n)$-invariant Kähler-Ricci flow with non-negative curvature
}

Bo Yang And Fangyang Zheng

\begin{abstract}
It is interesting to know the existence of the Kähler-Ricci flow on complete non-compact Kähler manifolds with non-negative holomorphic bisectional curvature. In this paper, we study $U(n)$ invariant Kähler-Ricci flow on $\mathbb{C}^{n}$ with non-negative curvature. Motivated by the recent work of $\mathrm{Wu}$ and the second named author [37], we also study examples of $U(n)$-invariant complete Kähler metrics on $\mathbb{C}^{n}$ with positive and unbounded curvature.
\end{abstract}

\section{Introduction}

2 Examples of $U(n)$-invariant Kähler metrics with positive curvature

2.1 Introduction

2.2 The results of $\mathrm{Wu}$ and the second named author

2.3 Proof of Propositions 2.1 and 2.2

2.3.1 Step 1: Set up and the generating function $p(x)$.

2.3.2 Step 2: Perturbation on $p(x)$ and estimates on $D$.

2.4 Various levels of positivity on the curvature

$3 U(n)$-invariant Kähler-Ricci flow on $\mathbb{C}^{n}$ with non-negative curvature

3.1 The $U(n)$-invariant Kähler-Ricci flow equation

3.2 Non-negativity of curvature is preserved

3.3 The asymptotic volume ratio is preserved 
4 Discussions on the existence of $U(n)$-invariant Kähler-Ricci flow

4.1 Theorem of Cabezas-Rivas and Wilking
4.2 Ricci flow on double covers with rotational symmetry

\subsection{Conditions that ensure the existence of Kähler-Ricci flow}

\section{Introduction}

One of the central questions around the study of complete non-compact positively curved Kähler manifolds is the uniformization conjecture whose most general form is due to Yau [41]. The conjecture states that any complete noncompact Kähler manifolds with positive holomorphic bisectional curvature is biholomorphic to complex Euclidean space. Some related questions are also asked by Greene and $\mathrm{Wu}$ [16] and Siu [36]. While this conjecture itself is still wide open, there are many progress in the past decades; see [7-9, $11,12,16,22,24,33,34]$ and references therein. In particular, the Ricci flow, introduced by Hamilton [17] in 1982, proves to be a powerful tool to study the uniformization problem since Shi's work [33, 34]. The best results obtained to date is the theorem of Chau and Tam [7] which says that a complete non-compact Kähler manifold with non-negative bisectional curvature is biholomorphic to complex Euclidean space if it has bounded curvature and Euclidean volume growth. All the important progress along the uniformization problem via the Ricci flow approach assume upper bounds of curvatures since they all rely on the long-time existence theorem of Kähler-Ricci flow proved by Shi [33, 34], which in turn is based on Shi's short-time existence result of Ricci flow on complete manifolds with bounded curvatures [32].

So far, all the available examples of complete Kähler metrics with positive bisectional curvature are constructed on $\mathbb{C}^{n}$ with $U(n)$-symmetry, see $[4,5,21,37]$. In particular, it was observed by $\mathrm{Wu}$ and the second named author [37] that there are many examples of Kähler metrics with positive but unbounded bisectional curvature. In general, it is unknown whether one 
can prove an existence theorem of Kähler-Ricci flow on any complete Kähler manifold with positive holomorphic bisectional curvature (without assuming boundedness of the curvature). Such a result, if exists, could be very helpful to study uniformization problem for Kähler manifolds with positive but unbounded curvature. For the Riemannian Ricci flow, the recent exciting work of Cabezas-Rivas and Wilking [3] proved a short-time existence theorem for any complete non-compact manifold with non-negative complex sectional curvature.

Those examples of Kähler metrics with positive bisectional curvature on $\mathbb{C}^{n}$ with $U(n)$-symmetry motivate us to consider the following question:

Question 1.1. Starting with any complete $U(n)$-invariant Kähler metric on $\mathbb{C}^{n}$ with non-negative holomorphic bisectional curvature, does there always exist a complete solution to the Kähler-Ricci flow with $U(n)$ symmetry?

In view of Cabezas-Rivas and Wilking's theorem [3], which assumes the non-negative complex sectional curvature, it is also interesting to understand $U(n)$-invariant Kähler metrics on $\mathbb{C}^{n}$ with different levels of non-negativity of curvatures. (E.g., non-negative complex sectional curvature, non-negative complex curvature operator.) The recent work of $\mathrm{Wu}$ and the second named author [37] developed a systematic way to construct $U(n)$-invariant complete Kähler metrics on $\mathbb{C}^{n}$ with positive bisectional curvature. In particular, they obtain some sufficient and necessary algebraic conditions on curvatures to characterize different levels of positive curvatures. The first result of our paper is a further development of their theory. We use a perturbation method to demonstrate that one can perturb some $U(n)$-invariant Kähler metrics on $\mathbb{C}^{n}$ with positive sectional curvature along any sequence going to infinity to obtain Kähler metrics with unbounded curvature while preserving the positivity of sectional curvatures. In particular, we show:

Proposition 1.2. Fix $n>2$, for any integer $1<k \leq n$, there exists a complete Kähler metric with positive and unbounded sectional curvature on $\mathbb{C}^{n}$ with $r^{-2 n+2 k} \int_{B(O, r)} \sigma_{k}$ unbounded when $r$ tends to infinity. Here with $\sigma_{k}$ is the kth elementary symmetric function of the Riemannian Ricci tensor and $B(O, r)$ is the geodesic ball of radius $r$ centered at some fixed point $O$.

It is interesting to compare it with the following result of Petrunin [30]. In fact, Proposition 1.2 is related to a question of Yau (See p. 278, Problem 9, $[40])$. 
Theorem 1.3 Petrunin [30]. Let $\left(M^{n}, g\right)$ be a complete Riemannian manifold with non-negative sectional curvature, then for any $O \in M$ and $r>0$, there exists a constant $c(n)$ which depends only on $n$ such that

$$
r^{2-n} \int_{B(O, r)} R d \operatorname{Vol}(g) \leq c(n),
$$

where $R$ is the scalar curvature of $\left(M^{n}, g\right)$.

Recall that Chen [10] proved that the non-negativity of the scalar curvature is preserved for any complete solution to the Ricci flow. Motivated by the new cut-off techniques developed in [10], we show that any complete Kähler-Ricci flow on $\mathbb{C}^{n}$ with $U(n)$-symmetry preserves various levels of non-negative curvatures. In general it is known that Kähler-Ricci flow preserves non-negativity of holomorphic bisectional curvature on compact manifolds [1,23] or complete manifolds with bounded curvature [33]. The point here is that by assuming the $U(n)$-symmetry we do not require any upper bounds on curvatures.

Theorem 1.4. Let $g(t)$ be a complete solution of Kähler-Ricci flow on $\mathbb{C}^{n}$ with $U(n)$-symmetry for $t \in[0, T]$, if the holomorphic bisectional curvature (sectional curvature, or complex curvature operator) of the initial metric $g(0)$ is non-negative, so is that of $g(t)$ for any $t \in[0, T]$. Furthermore, if $g(0)$ has holomorphic bisectional curvature (sectional curvature, or complex curvature operator) positive somewhere, then $g(t)$ has positive bisectional curvature (sectional curvature, or complex curvature operator) on $\mathbb{C}^{n} \times(0, T]$.

In fact, our method shows that any non-negative curvature conditions which lies between non-negative sectional curvature and non-negative complex curvature operator is always preserved by complete Kähler-Ricci flow on $\mathbb{C}^{n}$ with $U(n)$-symmetry. It is worth noting that in general, the nonnegative sectional curvature may not be preserved by Ricci flow on noncompact manifolds [25]. This motivates us to prove the following theorem. (See Section 2.4 for our definition on positive (non-negative) complex sectional curvature on general Kähler manifolds.)

Theorem 1.5. Let $g$ be a complete $U(n)$-invariant Kähler metric on $\mathbb{C}^{n}$. If $g$ has positive (non-negative) sectional curvature everywhere, then it will have positive (non-negative) complex sectional curvature everywhere.

Recall that it was shown in [28] that non-negative complex sectional curvature is preserved along Ricci flow on closed manifolds. Such an invariant curvature condition under Ricci flow is useful in the proof of the differentiable 
sphere theorem due to Brendle and Schoen [2]. Also Theorem 1.5 demonstrates a somewhat surprising rigidity phenomenon for $U(n)$-invariant Kähler metrics on $\mathbb{C}^{n}$ with positive curvature.

The asymptotic volume ratio (AVR) is another interesting quantity whose invariance under the Ricci flow was first studied by Hamilton [19]. There has been various generalizations of Hamilton's result in the context of Ricci flow and Kähler-Ricci flow, see [11, 12, 27, 31, 33, 42].

Theorem 1.6. Let $g(t)$ be a complete $U(n)$-invariant Kähler-Ricci flow on $\mathbb{C}^{n} \times[0, T]$ where $g(0)$ is of non-negative holomorphic bisectional curvature, then AVR of $\left(\mathbb{C}^{n}, g(t)\right)$ is constant on $[0, T]$. In fact, for any $t \in[0, T]$

$$
\lim _{s \rightarrow+\infty} \frac{V_{t}\left(B_{t}(O, s)\right)}{V_{0}\left(B_{0}(O, s)\right)}=1,
$$

where $B_{t}(O, s)$ denotes the geodesic ball of radius $s$ for the metric $g(t)$ centered at the origin.

Of course it is desirable if one can answer Question 1.1 affirmatively. Kähler-Ricci flow equation on $\mathbb{C}^{n}$ with $U(n)$-symmetry can be reduced to a non-linear equation of fast diffusion type. In the case of $n=1$ such equations have been studied extensively in the context of partial differential equations, and we refer the readers to check the review paper [20] and reference therein. However, in higher dimensions it seems very hard to solve such equations directly due to the high non-liearities involved. In the last part of the paper we study the Riemannian Ricci flow constructed by Cabezas-Rivas and Wilking [3] with the initial metric being a $U(n)$-invariant Kähler metric with non-negative complex sectional curvature on $\mathbb{C}^{n}$. Note that by Theorem 1.5 we only need to assume non-negative sectional curvature. Recall that Cabezas-Rivas and Wilking constructed such a Ricci flow after obtaining some delicate estimates on curvature evolution of the Ricci flows emanating from a sequence of double covers which converges to the original manifold in the sense of Cheeger-Gromov. Such double covers, obtained by gluing two copies of geodesic balls in the original manifold with increasing radii after identifying the boundary and perturbing the inner region nearby, are topologically spheres. Moreover, they are endowed with metrics with nonnegative complex sectional curvature. Ricci flows on those closed manifolds instantaneously evolve their curvatures into positive complex sectional curvatures (p. 6 in [3]), thus destroy the Kähler structures even when the initial metrics are Kähler on some open sets. Apriori it is not clear if one can get a complete Kähler-Ricci flow after taking limits on those closed Ricci flows. 
In the last section of this paper, we are able to show Cabezas-Rivas and Wilking's Ricci flow is indeed a Kähler-Ricci flow under some extra technical assumptions. We believe that those assumptions could be improved by more refined analysis on the curvature evolution for the Ricci flow on those double covers. We plan to study the existence of complete Kähler-Ricci flow with positive curvature in a general context in future works.

The paper is organized as follows: In Section 2, we use a perturbation method to construct examples of Kähler metrics with positive sectional curvature and unbounded curvature. Various levels of positivity on the curvature for $U(n)$-invariant Kähler metrics are discussed in Section 2.4. In particular, we prove Theorem 1.5. In Section 3 we prove that any complete Kähler-Ricci flow on $\mathbb{C}^{n}$ with $U(n)$-symmetry preserves non-negativity of various levels of curvature and the AVR. In the last section, we discuss the Ricci flow constructed by Cabezas-Rivas and Wilking [3] with the initial metric being $U(n)$-invariant Kähler metrics with non-negative sectional curvature on $\mathbb{C}^{n}$. We end up with an existence theorem for Kähler-Ricci flow with $U(n)$-symmetry under extra assumptions.

\section{Examples of $U(n)$-invariant Kähler metrics with positive curvature}

\subsection{Introduction}

Let $\mathcal{M}_{n}\left(\mathcal{N}_{n}, \mathcal{K}_{n}\right)$ denote the space of all $U(n)$-invariant complete Kähler metrics on $\mathbb{C}^{n}$ with positive bisectional curvature (positive sectional curvature, positive complex curvature operator). See Section 2.4 for the definition of complex curvature operator on Kähler manifolds. We will show the following theorems.

Proposition 2.1. There are examples in $\mathcal{N}_{n}$ and $\mathcal{K}_{n}$ with unbounded curvature.

It is shown in [37] that examples of metrics with unbounded curvature in $\mathcal{M}_{n}$ can be easily constructed. In fact, their results implies that one can perturb any metric in $\mathcal{M}_{n}$ such that the new one has its scalar curvature blowing up with any given rate along a prescribed sequence along the infinity. However, if we require stronger restrictions on the positivity of the curvature, more careful analysis on the perturbation are needed. In fact, we construct examples in Proposition 2.1 by perturbing a particularly chosen metric in $\mathcal{K}_{n}$, although we believe that such a choice should not be essential. 
As an application of the perturbation argument developed in the proof of Proposition 2.1, we prove

Proposition 2.2. Fix any $n>2$. For any integer $2 \leq k \leq n$, there exists a complete Kähler metric in $\mathcal{N}_{n}$ such that $r^{-2 n+2 k} \int_{B(O, r)} \sigma_{k}$ is unbounded when $r$ tends to infinity. Here $\sigma_{k}$ denotes the kth elementary symmetric function of the eigenvalues of the Ricci tensor and $B(O, r)$ is the geodesic ball of radius $r$ centered at a fixed point $O$.

This problem is related to a question raised by Yau [40] (see p. 278, Problem 9) as a generalization of the Cohn-Vossen inequality in high dimensions. Originally it is stated for manifolds with non-negative Ricci curvature. Recently Yau (see [39]) suggested that the original conjecture should be modified by assuming Riemannnian manifolds with non-negative sectional curvature and Kähler manifolds with non-negative bisectional curvature (In the latter case, the Ricci form of the Kähler metric is used instead). Note that, if one only requires non-negative Ricci curvature as in Yau's original question, then Proposition 2.2 can be proved by applying the characterization of Kähler metrics in $\mathcal{M}_{n}$ developed in [37], see [39] for details. In Section 2.3, we are able to construct examples in $\mathcal{N}_{n}$ satisfying the conclusion of Proposition 2.2 with a perturbation argument. The essential reason is that we can perturb some metric from $\mathcal{N}_{n}$ to adjust the radial curvature along a sequence of points tending to the infinity. Note that each eigenvalue of the Ricci tensor of a Kähler manifold is of multiplicity 2, and when $k>1$, one of summands of $\sigma_{k}$ contains the square of radial curvature. A careful choice of the perturbation function will allow us to prescribe $\sigma_{k}$ and get $r^{-n+2 k} \int_{B(r)} \sigma_{k}$ unbounded when $r$ is large.

\subsection{The results of $\mathrm{Wu}$ and the second named author}

In this section, we collect the main results from the recent work of $\mathrm{Wu}$ and the second author [37] where a systematic treatment of $U(n)$-invariant complete Kähler metrics on $\mathbb{C}^{n}$ with positive bisectional curvature was developed.

We will follow the notations in [37]. Let $z=\left(z_{1}, \ldots, z_{n}\right)$ be the standard coordinate on $\mathbb{C}^{n}$ and $r=|z|^{2}$. A $U(n)$-invariant Kähler metric on $\mathbb{C}^{n}$ has the Kähler form

$$
\omega=\frac{\sqrt{-1}}{2} \partial \bar{\partial} P(r),
$$


where $P \in C^{\infty}[0,+\infty)$. Under the local coordinates, the metric has components:

$$
g_{i \bar{j}}=f(r) \delta_{i j}+f^{\prime}(r) \bar{z}_{i} z_{j}
$$

We further denote:

$$
f(r)=P^{\prime}(r), \quad h(r)=(r f)^{\prime}
$$

It can be checked that the from $\omega$ will give a complete Kähler metric on $\mathbb{C}^{n}$ if and only if

$$
f>0, \quad h>0, \quad \int_{0}^{+\infty} \frac{\sqrt{h}}{\sqrt{r}} d r=+\infty .
$$

Now if we compute the components of the curvature tensor at $\left(z_{1}, 0, \ldots\right.$, $0)$ under the orthonormal frame $\left\{e_{1}=\frac{1}{\sqrt{h}} \partial_{z_{1}}, e_{2}=\frac{1}{\sqrt{f}} \partial_{z_{2}}, \ldots, e_{n}=\frac{1}{\sqrt{f}} \partial_{z_{n}}\right\}$, then denote by $A, B, C$, respectively:

$$
\begin{aligned}
& A=R_{1 \overline{1} 1 \overline{1}}=-\frac{1}{h}\left(\frac{r h^{\prime}}{h}\right)^{\prime}, \quad B=R_{1 \overline{1} i \bar{i}}=\frac{f^{\prime}}{f^{2}}-\frac{h^{\prime}}{h f}, \\
& C=R_{i \bar{i} i \bar{i}}=2 R_{i \bar{i} \bar{j} \bar{j}}=-\frac{2 f^{\prime}}{f^{2}}
\end{aligned}
$$

where we assume $2 \leq i \neq j \leq n$. It is easy to check that all other components of the curvature tensor are zero.

Theorem 2.3 Characterizing $\mathcal{M}_{n}, \mathcal{N}_{n}$, and $\mathcal{K}_{n}$ by $A B C D$ functions [37]. Suppose $n \geq 2$ and $h$ is a smooth positive function on $[0,+\infty)$ satisfying (2.4), then(2.1) gives a complete Kähler metric in $\mathcal{M}_{n}$ if and only if $A, B, C$ are positive. Moreover, it is in $\mathcal{N}_{n}$ iff $D \doteq A C-B^{2}>0$, and in $\mathcal{K}_{n}$ iff $D_{n} \doteq \frac{n}{2(n-1)} A C-B^{2}>0$.

If we define a smooth function $\xi$ on $[0,+\infty)$ by

$$
\xi(r)=-\frac{r h^{\prime}(r)}{h},
$$

then $h$ determines $\xi$ uniquely. On the other hand, note that $\xi$ determines $h$ by $h(r)=h(0) \mathrm{e}^{\int_{0}^{r} \frac{\xi(t)}{t} d t}$. Hence $\xi$ determines $\omega$ up to a constant multiple. The following observation in [37] reveals that the space $\mathcal{M}_{n}$ is actually quite large. 
Theorem 2.4 Characterizing $\mathcal{M}_{n}$ by the $\xi$ function [37]. Suppose $n \geq 2$ and $h$ is a smooth positive function on $[0,+\infty)$, then the form defined by (2.1) gives a complete Kähler metric with positive bisectional curvature on $\mathbb{C}^{n}$ if and only if $\xi$ defined by (2.6) satisfying

$$
\xi(0)=0, \quad \xi^{\prime}>0, \quad \xi<1
$$

$\mathrm{Wu}$ and Zheng [37] also introduced another function $F$ in the following way: First let $x=\sqrt{r h}$, and define a non-negative function $y$ of $r$ by

$$
y(0)=0, \quad x^{\prime 2}+y^{\prime 2}=\frac{h}{4 r}, \quad y^{\prime}>0
$$

One can check that $x(r)$ is strictly increasing, so we get a smooth function $F$ on $\left[0, x_{0}\right)$ by $y=F(x)$. Here, $x_{0} \leq \infty$ is determined by

$$
x_{0}^{2}=\lim _{r \rightarrow+\infty} r h=h(1) \mathrm{e}^{\int_{1}^{+\infty} \frac{1-\xi}{r} d r}
$$

Extending $F$ to $\left(-x_{0}, x_{0}\right)$ by letting $F(x)=F(-x)$, one can check that $F$ is a smooth, even function on $|x|<x_{0}$. Starting with such an $F$ satisfying certain conditions, one can recover the metric $\omega$ in a geometric way. See Section 5 in [37] for details. This result is summarized as the following theorem.

Theorem 2.5 Characterizing $\mathcal{M}_{\boldsymbol{n}}$ by the $F$ function [37]. For any $n \geq 1$, there is a one one correspondence between the set $\mathcal{M}_{n}$ and the set $\mathcal{F}$ of smooth, even function $F(x)$ defined on $\left(-x_{0}, x_{0}\right)$ satisfying

$$
F(0)=0, \quad F^{\prime \prime}>0, \quad \lim _{x \rightarrow x_{0}} F(x)=+\infty
$$

All the above results can be generalized to the case of $U(n)$-invariant complete Kähler metrics on $\mathbb{C}^{n}$ with non-negative bisectional curvature [39].

\subsection{Proof of Propositions 2.1 and 2.2}

2.3.1. Step 1: Set up and the generating function $\boldsymbol{p}(\boldsymbol{x})$. Now assume $F:\left[0, x_{0}\right) \rightarrow[0, \infty)$ is a smooth strictly convex function where 
$0<x_{0} \leq+\infty, F(0)=F^{\prime}(0)=0$, and $F\left(x_{0}\right)=+\infty$. Write

$$
p(x)=\sqrt{1+\left(F^{\prime}(x)\right)^{2}}, \quad v(x)=\int_{0}^{x} 2 \tau p(\tau) d \tau .
$$

We will call $p(x)$ the generating function for the metric in $\mathcal{M}_{n}$. One can rewrite $\mathrm{ABC}$ in terms of $p(x)$ :

$$
\begin{aligned}
A & =\frac{F^{\prime} F^{\prime \prime}}{2 x\left(1+\left(F^{\prime}\right)^{2}\right)^{2}}=\frac{p^{\prime}}{2 x p^{3}} . \\
B & =\frac{1}{v^{2}}\left(x^{2}-\frac{v}{\sqrt{1+\left(F^{\prime}(x)\right)^{2}}}\right)=\frac{1}{v^{2}}\left(x^{2}-\frac{v}{p}\right) . \\
C & =\frac{2}{v^{2}}\left(v-x^{2}\right) .
\end{aligned}
$$

The problem of characterizing the space $\mathcal{N}_{n}$ or $\mathcal{K}_{n}$ can be reduced to the following problem:

Question 2.6. Let $0<x_{0} \leq \infty$, and let $p(x)$ be a smooth strictly increasing function on $\left[0, x_{0}\right)$ with $p(0)=1, p^{\prime}(0)=0, p^{\prime \prime}(0)>0$ and $\int_{0}^{x_{0}} p(\tau) d \tau=$ $+\infty$. Can we find $p(x)$ such that

$$
\frac{p^{\prime} v^{2}}{x p^{3}}\left(v-x^{2}\right)>\left(x^{2}-\frac{v}{p}\right)^{2} \quad \text { or } \quad \frac{n}{2 n-2} \frac{p^{\prime} v^{2}}{x p^{3}}\left(v-x^{2}\right)>\left(x^{2}-\frac{v}{p}\right)^{2}
$$

holds for all $x \in\left(0, x_{0}\right)$ ? Here $v(x)=\int_{0}^{x} 2 \tau p(\tau) d \tau$. Note that $\mathcal{N}_{n}$ and $\mathcal{K}_{n}$ are the same when $n=2$. In general the second named author [43] proved that any complete Kähler surface with positive sectional curvature must have positive complex curvature operator, See Section 2.4 for more discussion.

2.3.2. Step 2: Perturbation on $p(x)$ and estimates on $D$. First we recall the following examples in [37].

Example 2.7 [37]. $\xi=\frac{c r}{1+r}$ where $r \in[0,+\infty)$. Then for any $0<c<1$ the corresponding metric lies in $\mathcal{M}_{n}$ and has maximal volume growth; it lies in $\mathcal{K}_{n}$ if $0<c \leq \frac{1}{2}$ while it dose not have positive sectional curvature for any $\frac{1}{2}<c<1$. 
Let us fix $c=\frac{1}{2}$. A routine calculation shows:

$$
A=\frac{1}{2(1+r)^{\frac{3}{2}}}, \quad B=\frac{1}{4(1+r)}, \quad C=\frac{1}{2 \sqrt{1+r}}, \quad D=\frac{3}{16(1+r)^{2}} .
$$

$$
\begin{gathered}
s=\int_{0}^{r} \frac{1}{2 \sqrt{r(1+r)^{\frac{1}{2}}}} d r \sim r^{\frac{1}{4}} . \\
p=\frac{1+r}{1+\frac{r}{2}}, \quad p^{\prime}(x)=\frac{r^{\frac{1}{2}}(1+r)^{\frac{5}{4}}}{\left(1+\frac{r}{2}\right)^{3}}, \quad v=2(\sqrt{1+r}-1) . \\
x^{2}=\frac{r}{\sqrt{1+r}}, \quad r=\frac{x^{4}+\sqrt{x^{8}+4 x^{4}}}{2}
\end{gathered}
$$

Our goal is to perturb the function $p(x)$ to produce a Kähler metric in $\mathcal{N}_{n}$ with unbounded curvature along a sequence of points tending to infinity.

Define

$$
\widetilde{p}=p+\Phi, \quad E=\int_{0}^{x} 2 \tau \Phi(\tau) d \tau
$$

Here we assume that $\Phi$ is non-negative non-decreasing function on $[0,+\infty)$ and vanishes in a small neighborhood of 0 . These conditions ensure that the new function $\widetilde{p}(x)$ will be a generating function for some metric in $\mathcal{M}_{n}$. Note that we have $\widetilde{v}=\int_{0}^{x} 2 \tau \widetilde{p}(\tau) d \tau=v+E$, so it is straightforward to get the curvature components of the new metric generated by $\widetilde{p}$ :

$$
\begin{aligned}
\widetilde{A} & =\frac{\widetilde{p}^{\prime}}{2 x \widetilde{p}^{3}}=\frac{p^{\prime}(x)+\Phi^{\prime}(x)}{2 x(p+\Phi)^{3}} . \\
\widetilde{B} & =\frac{1}{\widetilde{v}^{2}}\left(x^{2}-\frac{\widetilde{v}}{\widetilde{p}}\right)=\frac{x^{2}}{(v+E)^{2}}-\frac{1}{(v+E)(p+\Phi)} \\
\widetilde{C} & =\frac{2}{\widetilde{v}^{2}}\left(\widetilde{v}-x^{2}\right)=\frac{2}{v+E}-\frac{2 x^{2}}{(v+E)^{2}} .
\end{aligned}
$$

The point of Step 2 is to estimate $\widetilde{D} \doteq \widetilde{A} \widetilde{C}-\widetilde{B}^{2}$ via the following lemma.

Lemma 2.8. There exists $\delta_{0}>0$ and two constants $C\left(\delta_{0}\right)$ and $x_{0}$ both only depending on $\delta_{0}$ such that for any $0<\delta \leq \delta_{0}$ and any smooth increasing 
function $\Phi(x)$ defined on $[0,+\infty)$ with $\Phi=0$ in a small neighborhood of 0 and

$$
\lim _{x \rightarrow+\infty} \Phi(x)=\delta, \quad \int_{0}^{+\infty} \Phi^{\prime}(\tau) \tau^{2} d \tau \leq C\left(\delta_{0}\right) \delta,
$$

we always have $\widetilde{D}>0$ for any $x>x_{0}$.

Assume that we have proved Lemma 2.8, then we pick any $0<\delta<\delta_{0}$ and any function $\Phi$ satisfying the assumption in Lemma 2.8. Consider $\widetilde{D}$ inside the ball with $0 \leq x \leq 2 x_{0}$. Since $\widetilde{D}$ converges to $D>0$ uniformly on compact sets $\overline{B\left(O, 2 x_{0}\right)}$ as $\delta \rightarrow 0$, shrinking $\delta$ if necessary, we conclude that there exists a function $\Phi(x)$ defined on $[0,+\infty)$ such that

$$
\lim _{x \rightarrow+\infty} \Phi(x)=\delta, \quad \int_{0}^{+\infty} \Phi^{\prime}(\tau) \tau^{2} d \tau \leq C\left(\delta_{0}\right) \delta,
$$

such $\widetilde{D}>0$ for this particular $\Phi(x)$ inside the ball with $0 \leq x \leq 2 x_{0}$. Therefore, $\widetilde{D}>0$ everywhere and $\widetilde{p}(x)$ generates a metric with positive sectional curvature.

Proof of Lemma 2.8. Note that:

$$
\begin{aligned}
\widetilde{D}-D= & (A+\widetilde{A}-A)(C+\widetilde{C}-C)-(B+\widetilde{B}-B)^{2}-\left(A C-B^{2}\right) \\
= & (\widetilde{A}-A) C+(\widetilde{C}-C) A+(\widetilde{A}-A)(\widetilde{C}-C) \\
& -2 B(\widetilde{B}-B)-(\widetilde{B}-B)^{2}
\end{aligned}
$$

Step 1 of Proof of Lemma 2.8: estimating $\widetilde{A}-A$.

It follows from (2.11) and (2.20) that

$$
\widetilde{A}-A=\frac{\Phi^{\prime}}{2 x(p+\Phi)^{3}}-\frac{p^{\prime}\left(3 p^{2} \Phi+3 p \Phi^{2}+\Phi^{3}\right)}{2 x p^{3}(p+\Phi)^{3}} .
$$

Then we know $\widetilde{A}-A \geq 0$ at points where

$$
\Phi^{\prime}(x) \geq \Phi \frac{p^{\prime}(x)}{p^{3}}\left(\Phi^{2}+3 \Phi p+3 p^{2}\right) .
$$

Otherwise, we have

$$
-\frac{p^{\prime}(x)}{2 x p^{6}}\left(\delta^{3}+6 \delta^{2}+12 \delta\right) \leq \widetilde{A}-A \leq 0,
$$

where we use $1 \leq p \leq 2$. 
Note that $\frac{P^{\prime}(x)}{2 x p^{3}} \sim O\left(\frac{1}{r^{\frac{3}{2}}}\right)$. we conclude that there exists $x_{0}$ and $c(\delta)$ only depends on $\delta$ such that

$$
\text { If } \widetilde{A}-A \leq 0 \text {, then }-\frac{c(\delta)}{r^{\frac{3}{2}}} \leq \widetilde{A}-A \leq 0 \text { whenever } x \geq x_{0} \text {. }
$$

Step 2 of Proof of Lemma 2.8: estimating $\widetilde{C}-C$.

It follows from (2.13) and (2.22) that

$$
\widetilde{C}-C=\frac{-2 E}{v(v+E)}+2 x^{2} \frac{E^{2}+2 v E}{v^{2}(v+E)^{2}} .
$$

Note that $v \leq 2 \sqrt{r}$ and $x^{2} \leq \sqrt{r}$.

$$
\frac{2 E}{v(v+E)}+x^{2} \frac{E^{2}+2 v E}{v^{2}(v+E)^{2}} \leq \frac{\delta}{2 \sqrt{r}}+\frac{2 \delta+\delta^{2}}{4 \sqrt{r}} .
$$

Similarly we conclude that there exists $c(\delta)$ only depends on $\delta$ such that

$$
|\widetilde{C}-C| \leq \frac{c(\delta)}{\sqrt{r}}
$$

Step 3 of Proof of Lemma 2.8: estimating $\widetilde{B}-B$.

It follows from (2.12) and (2.21) that

$$
\widetilde{B}-B=-x^{2} \frac{E^{2}+2 E v}{(v+E)^{2} v^{2}}+\frac{E p+E \Phi+v \Phi}{v p(v+E)(p+\Phi)} .
$$

Now plug in $v=2(\sqrt{1+r}-1), x^{2}=\frac{r}{\sqrt{r+1}}$ and $p=\frac{1+r}{1+\frac{r}{2}}$. Denote $\widetilde{B}-$ $B=I_{1}-I_{2}$ where

$$
\begin{aligned}
& I_{1}=\frac{r}{\sqrt{r+1}} \frac{E^{2}+2 E \cdot 2(\sqrt{r+1}-1)}{[2(\sqrt{1+r}-1)+E]^{2}[2(\sqrt{r+1}-1)]^{2}}, \\
& I_{2}=\frac{E\left(\frac{1+r}{1+\frac{r}{2}}\right)+E \Phi+2(\sqrt{r+1}-1) \Phi}{2(\sqrt{r+1}-1)[2(\sqrt{r+1}-1)+E]\left(\frac{1+r}{1+\frac{r}{2}}\right)\left[\frac{1+r}{1+\frac{r}{2}}+\Phi\right]} .
\end{aligned}
$$

The following claim is crucial in Step 3 of Proof of Lemma 2.8. 
Claim 2.9.

$$
\begin{gathered}
\lim _{r \rightarrow+\infty} \sqrt{r} I_{1}=\lim _{r \rightarrow+\infty} \sqrt{r} I_{2}=\frac{\delta^{2}+4 \delta}{4(2+\delta)^{2}}, \\
r\left|I_{1}-I_{2}\right| \leq c(\delta),
\end{gathered}
$$

for constant $c(\delta)$ and $x \geq x_{0}$ where $x_{0}$ only depends on $\delta$.

Proof of Claim 2.9. (2.36) is straightforward if we note

$$
\lim _{r \rightarrow+\infty} \frac{E}{\sqrt{r}}=\lim _{x \rightarrow+\infty} \Phi(x)=\delta .
$$

Introduce:

$$
\begin{aligned}
I_{3} \doteq & r\left[E^{2}+2 E \cdot 2(\sqrt{r+1}-1)\right] 2(\sqrt{r+1}-1)\left(\frac{1+r}{1+\frac{r}{2}}\right) \\
& \times[2(\sqrt{r+1}-1)+E]\left[\frac{1+r}{1+\frac{r}{2}}+\Phi\right], \\
I_{4} \doteq & \sqrt{1+r}[E+2(\sqrt{r+1}-1)]^{2}[2(\sqrt{r+1}-1)]^{2} \\
& \times\left[E\left(\frac{1+r}{1+\frac{r}{2}}\right)+E \Phi+2(\sqrt{r+1}-1) \Phi\right] .
\end{aligned}
$$

To prove (2.37) we will show there exists constants $c(\delta)$ and $x_{0}$ which only depends on $\delta$

$$
\frac{\left|I_{3}-I_{4}\right|}{r^{\frac{5}{2}}} \leq c(\delta)
$$

for any $x \geq x_{0}$.

Note the fact that $\sqrt{r+1}-1=\sqrt{r}-C_{1}, \frac{1+r}{1+\frac{r}{2}}=2-\frac{C_{2}}{r}$, and $\sqrt{1+r}-$ $\sqrt{r}=\frac{C_{3}}{\sqrt{r}}$, where $C_{1}, C_{2}$ and $C_{3}$ are bounded functions of $r$.

$$
\begin{aligned}
I_{3}= & r\left[E^{2}+4 E \sqrt{r}-2 C_{1} E\right]\left[2 \sqrt{r}-2 C_{1}\right] \\
& \times\left[2-\frac{C_{2}}{r}\right]\left[2 \sqrt{r}+E-2 C_{1}\right]\left[2+\Phi-\frac{C_{2}}{r}\right], \\
I_{4}= & {\left[\sqrt{r}+\frac{C_{3}}{\sqrt{r}}\right]\left[E+2 \sqrt{r}-2 C_{1}\right]^{2}\left[2 \sqrt{r}-2 C_{1}\right]^{2} } \\
& \times\left[2 E+E \Phi+2 \sqrt{r} \Phi-\frac{C_{2}}{r} E-2 C_{1} \Phi\right] .
\end{aligned}
$$


It is straightforward to check that:

$$
\frac{\left|I_{3}-I_{4}\right|}{r^{\frac{5}{2}}}=16(\delta+2)(E-\sqrt{r} \Phi)+c(\delta),
$$

where $c(\delta)$ only depends on $\delta$.

A further integration of parts shows:

$$
\begin{aligned}
E-\sqrt{r} \Phi & =\int_{0}^{x} 2 \tau \Phi(\tau) d \tau-\sqrt{r} \Phi \\
& =x^{2} \Phi(x)-\int_{0}^{x} \Phi^{\prime}(x) \tau^{2} d \tau-\sqrt{r} \Phi \\
& =O\left(\frac{1}{\sqrt{r}}\right) \Phi(x)-\int_{0}^{x} \Phi^{\prime}(\tau) \tau^{2} d \tau
\end{aligned}
$$

To sum up, we need

$$
\int_{0}^{+\infty} \Phi^{\prime}(\tau) \tau^{2} d \tau \leq c(\delta)
$$

to ensure that $|\widetilde{B}-B| \leq \frac{C(\delta)}{r}$ for $r$ large. Claim 2.9 is proved.

To sum up, we proved Lemma 2.8 .

Now we are ready to prove both Propositions 2.1 and 2.2 by the perturbation method established above.

Proof of Proposition 2.1. Now that Lemma 2.8 is proved, it is easy to prescribe a suitable perturbation function $\Phi(x)$ to prove Proposition 2.1.

Define the following function $\eta$ on $[0,+\infty)$ with $\alpha$ and $\beta$ to be determined.

$$
\eta(x)= \begin{cases}2^{\alpha} & x \in\left[2,2+\left(\frac{1}{2}\right)^{\beta}\right] \\ 3^{\alpha} & x \in\left[3,3+\left(\frac{1}{3}\right)^{\beta}\right] \\ \vdots & \vdots \\ 0 & x \in[0,+\infty) \backslash\left(\cup_{l \geq 2}\left[l, l+\left(\frac{1}{l}\right)^{\beta}\right]\right) .\end{cases}
$$

Set $\alpha>1$ and $\beta>\alpha+3$, then it is easy to check.

$$
\int_{0}^{+\infty} \eta(\tau) d \tau<\infty, \quad \int_{0}^{x} \eta(\tau) \tau^{2} d \tau<\infty
$$


After a suitable smoothing of $\eta$, still denoted by $\eta$ for simplicity, choose

$$
\Phi^{\prime}(x)=\delta \frac{\eta(x)}{\int_{0}^{+\infty} \eta(\tau) d \tau} .
$$

Here we choose a sufficiently small $\delta \leq \delta_{0}$ where $\delta_{0}$ is defined in Lemma 2.8.

Note that

$$
\widetilde{A}=\frac{p^{\prime}(x)+\Phi^{\prime}(x)}{2 x(p+\Phi)^{3}} .
$$

will be unbounded along a sequence going to infinity. This will produce a metric in $\mathcal{N}_{n}$ with unbounded curvature. The proof of Proposition 2.1 is done since a similar perturbation estimates on $D_{n}$ works.

Proof of Proposition 2.2. Fix any fixed $2 \leq k \leq n$, it suffices to show that we can find $p(x)$ defined in Question 2.6 with $x_{0}=+\infty$ such that both $(2.14)$ and the following hold.

$$
\limsup _{x \rightarrow+\infty} \frac{1}{x^{2 n-2 k}} \int_{0}^{x} \frac{p^{2}}{p^{2}-1}\left[p^{\prime}(\tau)\right]^{2} \tau^{2(n-k)+1} d \tau=+\infty .
$$

Compare with the proof to Proposition 2.1, we only need to show: Fix any fixed $n>2$ and $2 \leq k \leq n$, we can find a smooth increasing function $\Phi(x)$ defined on $[0,+\infty)$ with $\Phi=0$ at a small neighborhood of 0 . such that there exists a constant $\delta$ small enough and $C$ independent of $\delta$ :

$$
\begin{aligned}
& \lim _{x \rightarrow+\infty} \Phi(x)=\delta, \quad \int_{0}^{+\infty} \Phi^{\prime}(\tau) \tau^{2} d \tau \leq C \delta, \\
& \limsup _{x \rightarrow+\infty} \frac{1}{x^{2 n-2 k}} \int_{0}^{x}\left[\Phi^{\prime}(\tau)\right]^{2} \tau^{2(n-k)+1} d \tau=+\infty .
\end{aligned}
$$

That could be done by assuming $\alpha+3<\beta<2 \alpha+2$ on the function $\eta$ defined in the proof of Proposition 2.1.

\subsection{Various levels of positivity on the curvature}

In this subsection, we will discuss the different levels of positivity for metrics in $\mathcal{M}_{n}$.

Start with an arbitrary Kähler manifold $\left(M^{n}, g\right)$ of complex dimension $n$. At any $p \in M$, the complexified tangent space $T=T_{p} M \otimes_{\mathbb{R}} \mathbb{C}$ splits as $V \oplus \bar{V}$, with $V \cong \mathbb{C}^{n}$ the space of all type $(1,0)$ tangent vectors. Extend the Riemannian curvature tensor $R$ of $g$ linearly over $\mathbb{C}$. The Kählerness of $g$ implies that the curvature operator of $g$, as a symmetric bilinear form 
on $\Lambda^{2} T=\Lambda^{2} V \oplus(V \otimes \bar{V}) \oplus \Lambda^{2} \bar{V}$, vanishes on the first and the third components of the right-hand side. It can be identified with the following Hermitian bilinear form $Q$ on $V \otimes \bar{V}$ defined by:

$$
Q(\xi, \bar{\eta})=\sum_{i, j, k, l=1}^{n} R_{i \bar{j} k \bar{l}} \xi_{i \bar{j}} \overline{\eta_{l \bar{k}}}
$$

where $R_{i \bar{j} k \bar{l}}$ are the components of $R$ under a unitary basis $\left\{e_{1}, \ldots, e_{n}\right\}$ of $V$, and with $\xi=\sum \xi_{i j} e_{i} \otimes \overline{e_{j}}$ and $\eta=\sum \eta_{i j} e_{i} \otimes \overline{e_{j}}$. We will call $Q$ the complex curvature operator of $g$.

For any $1 \leq k \leq n$, we will say that $\left(M^{n}, g\right)$ have $k$-positive curvature, or $Q$ is $k$-positive, if $Q(\xi, \bar{\xi})>0$ for any $0 \neq \xi \in V \otimes \bar{V}$ with $\operatorname{rank}(\xi) \leq k$. Here the rank of $\xi$ is defined to be the rank of the matrix $\left(\xi_{i \bar{j}}\right)$ under any basis $e$ of $V$. Similarly, one can define the notion of $k$-non-negative, $k$-negative, or $k$-non-positive.

So 1-positive means that $g$ has positive bisectional curvature, and $n$-positive means that $g$ has positive complex curvature operator. We point out that Kähler manifold $\left(M^{n}, g\right)$ has 2-positive curvature means exactly that $g$ has positive complex sectional curvature, namely, for any non-zero element $\sigma \in V \otimes \bar{V}$, it holds that $-R(\sigma, \bar{\sigma})>0$.

When $g$ has 2-positive (2-non-negative) curvature, its sectional curvature must be positive (or non-negative), but the converse may not be true in general, when the complex dimension $n>2$. (For $n=2$ it is proved in [43] that the positivity of sectional curvature is equivalent to the positivity of the complex curvature operator).

For our metric in $\mathcal{M}_{n}$, however, we will show that the 2-positivity is always equivalent to the positivity of the sectional curvature of $g$. In other words, any metric in $\mathcal{N}_{n}$ will have positive complex sectional curvature. This result gives an intuitive explanation why we are able to prove that nonnegative sectional curvature is preserved under $U(n)$-invariant Kähler-Ricci flow $\mathbb{C}^{n}$ in Section 3.2.

Theorem 2.10. Let $g$ be a complete $U(n)$-invariant Kähler metric on $\mathbb{C}^{n}$. If $g$ has positive (non-negative) sectional curvature everywhere, then it will have 2-positive (2-non-negative) curvature everywhere.

Proof of Theorem 2.10. Let us use the notations discussed in the early part of this section. Let $g \in \mathcal{N}_{n}$, we have $D=A C-B^{2}>0$ everywhere. Fix a point $z=\left(z_{1}, 0, \ldots, 0\right)$. We want to show that $Q(\xi, \bar{\xi})>0$ for any $\xi \neq 0$ with rank at most 2 . Under the unitary tangent frame $\left\{e_{1}, \ldots, e_{n}\right\}$ at $z$, the only 
non-zero components of the curvature tensor are $R_{i \bar{i} j \bar{j}}$. Denote it by $P_{i j}$, then $P_{11}=A, P_{1 i}=B, P_{i i}=C$, and $P_{i j}=\frac{1}{2} C$ for any $2 \leq i \neq j \leq n$.

For convenience, let us choose a new frame $\tilde{e}_{1}=\rho e_{1}, \tilde{e}_{i}=e_{i}$ for $2 \leq i \leq$ $n$, where $\rho=\frac{\sqrt{C}}{\sqrt{2 B}}$. Then under the new frame $\tilde{e}$, the only non-zero components of the curvature tensor are $\tilde{R}_{i \bar{i} j \bar{j}}=\tilde{P}_{i j}$, where

$$
\tilde{P}_{i j}=\frac{C}{2}\left(1+\delta_{i j}\right)+\delta_{i 1} \delta_{j 1} \frac{C}{2}\left(\frac{A C}{2 B^{2}}-2\right) .
$$

Now write $\xi=\sum_{i, j=1}^{n} \xi_{i j} \tilde{e}_{i} \bar{e}_{j}$. We have

$$
\begin{aligned}
\frac{2}{C} Q(\xi, \bar{\xi}) & =\frac{2}{C} \sum_{i, j=1}^{n} \tilde{P}_{i j} \xi_{i \bar{i}} \overline{\xi_{j \bar{j}}}+\frac{2}{C} \sum_{i \neq j} \tilde{P}_{i j}\left|\xi_{i \bar{i}}\right|^{2} \\
& =\left(\frac{A C}{2 B^{2}}-2\right)\left|\xi_{1 \overline{1}}\right|^{2}+\left|\sum_{i=1}^{n} \xi_{i \bar{i}}\right|^{2}+\sum_{i, j=1}^{n}\left|\xi_{i \bar{j}}\right|^{2}
\end{aligned}
$$

In particular, when $\xi_{1 \overline{1}}=0$ and $\xi \neq 0$, we have $Q(\xi, \bar{\xi})>0$. So scale $\xi$ if necessary, we may assume from now on that $\xi_{1 \overline{1}}=1$. Notice that any unitary change on the subframe $\left\{\tilde{e}_{2}, \ldots, \tilde{e}_{n}\right\}$ will not affect the components of the curvature tensor, so we may assume that the lower right $(n-1) \times(n-1)$ block of the matrix $\left(\xi_{i \bar{j}}\right)$ only have non-zero entries in its first two rows. In particular, only the first three elements on the diagonal line of $\left(\xi_{i \bar{j}}\right)$ might be non-zero. Let us denote the upper left $3 \times 3$ corner of $\left(\xi_{i \bar{j}}\right)$ by

$$
E=\left(\begin{array}{ccc}
1 & v_{1} & v_{2} \\
u_{1} & x & z \\
u_{2} & 0 & y
\end{array}\right)
$$

Note that by performing a unitary change of $\left\{e_{2}, e_{3}\right\}$ if necessary, we could make the $(3,2)$-entry zero. Since the trace of the matrix $\left(\xi_{i \bar{j}}\right)$ is $1+x+y$, we have

$$
\begin{aligned}
\frac{2}{C} Q(\xi, \bar{\xi}) & \geq\left(\frac{A C}{2 B^{2}}-2\right)+1+|u|^{2}+|v|^{2}+|x|^{2}+|y|^{2}+|z|^{2}+|1+x+y|^{2} \\
& =\left(\frac{A C}{2 B^{2}}-2\right)+1+f \\
& =\frac{1}{2}\left(\frac{A C}{B^{2}}-1\right)-\frac{1}{2}+f>-\frac{1}{2}+f,
\end{aligned}
$$


since $D=A C-B^{2}>0$. Here we wrote $|u|^{2}=\left|u_{1}\right|^{2}+\left|u_{2}\right|^{2}$ and $|v|^{2}=\left|v_{1}\right|^{2}+$ $\left|v_{2}\right|^{2}$. Since the rank of $\xi$ is at most 2 , we have

$$
\operatorname{det} E=x y+z u_{2} v_{1}-x u_{2} v_{2}-y u_{1} v_{1}=0 \text {. }
$$

Our goal is to show that $f \geq \frac{1}{2}$ for any $w=(u, v, x, y, z) \in \mathbb{C}^{7}$ with $\operatorname{det} E=$ 0 . Assume the contrary, namely, inf $f=c<\frac{1}{2}$. Take a sequence of points $\left\{w_{k}\right\}$ in $V=\{\operatorname{det} E=0\} \subset \mathbb{C}^{7}$ such that $f\left(w_{k}\right) \rightarrow a$. Since $f$ dominates the square of the Euclidean distance of $\mathbb{C}^{7},\left\{w_{k}\right\}$ is bounded, thus having a subsequence converging a point $w \in V$. We have $f(w)=c<\frac{1}{2}$. Let us fix such a point $w$, and we will derive a contradiction from this.

First notice that $x \neq 0$, since otherwise $f \geq|y|^{2}+|1+y|^{2} \geq \frac{1}{2}$. Similarly, $y \neq 0$. Next, notice that when $u_{2}=0$, we have $x=u_{1} v_{1}$, thus $|u|^{2}+$ $|v|^{2} \geq 2\left|u_{1} v_{1}\right|=2|x|$, which leads to

$$
\begin{aligned}
f & \geq 2|x|+|x|^{2}+|y|^{2}+|1+x+y|^{2} \\
& \geq 2|x|+|x|^{2}+\frac{1}{2}|1+x|^{2} \geq \frac{1}{2}
\end{aligned}
$$

contradicting with the assumption that $f(w)<\frac{1}{2}$. So we must have $u_{2} \neq 0$, and similarly, $v_{1} \neq 0$. Near the point $w, V$ is the smooth hypersurface in $\mathbb{C}^{7}$ given by the graph of

$$
z=x \frac{v_{2}}{v_{1}}+y \frac{u_{1}}{u_{2}}-\frac{x y}{u_{2} v_{1}}
$$

and $w$ is a local minimum point of the function $f$, now viewed as a function of $(u, v, x, y)$. The first order derivatives of $f$ are all zero at $w$, from these equations we get

$$
\begin{aligned}
& v_{1} \bar{v}_{2}=-x \bar{z}, \quad u_{2} \bar{u}_{1}=-y \bar{z}, \\
& \left|u_{2}\right|^{2}-\left|v_{2}\right|^{2}=\left|v_{1}\right|^{2}-\left|u_{1}\right|^{2}=|z|^{2}, \\
& 2|x|^{2}+x(1+\bar{y})+|z|^{2}+\left|u_{1}\right|^{2}=0 \\
& 2|y|^{2}+y(1+\bar{x})+|z|^{2}+\left|v_{2}\right|^{2}=0 .
\end{aligned}
$$

If $z=0$, then by (2.57), we have $v_{2}=u_{1}=0$, so $u_{2}=v_{1}=0$ by (2.58), a contradiction. So $z \neq 0$ at $w$. So again by (2.57) we know that $u_{1} v_{2} \neq 0$ at $w$ as well. Let us write $\alpha=v_{1} \bar{v}_{2}$ and $\beta=u_{2} \bar{u}_{1}$. Then we have $x=-\frac{\alpha}{\bar{z}}$ and $y=$ $-\frac{\beta}{\bar{z}}$. Plug them into (2.59) and (2.60) in the above, and write $a=\left|\frac{u_{2}}{v_{2}}\right|^{2}>1$ 
and $b=\left|\frac{v_{1}}{u_{1}}\right|^{2}>1$, note that $(2.58)$ we get

$$
z=(a+1) \bar{\alpha}+\bar{\beta}=\bar{\alpha}+(b+1) \bar{\beta} .
$$

Thus, $a \bar{\alpha}=b \bar{\beta}$, which is just $\alpha=\beta$ since (2.57). Hence $a=b$.

Let us write $\left|u_{1}\right|^{2}=\left|v_{2}\right|^{2}=\rho>0$. Then we have $\left|u_{2}\right|^{2}=\left|u_{1}\right|^{2}=a \rho$, and $|z|^{2}=(a-1) \rho>0$. Since $z=(a+2) \bar{\alpha}$, we get $(a-1) \rho=(a+2)^{2} a \rho^{2}$, so

$$
\rho=\frac{a-1}{a(a+2)^{2}}>0
$$

We also have $x=y=-\frac{1}{a+2}$. So at $w$, we have

$$
f=2 \rho+2 a \rho+(a-1) \rho+\frac{2}{(a+2)^{2}}+\left(1-\frac{2}{a+2}\right)^{2}=\frac{a^{3}+3 a^{2}-1}{a(a+2)^{2}} .
$$

On the other hand, since $w$ is on $V=\{\operatorname{det} E=0\}$, we have

$$
(a+2) \bar{\alpha}=-\frac{2}{a+2}\left(\frac{\bar{\alpha}}{a \rho}\right)-\frac{1}{(a+2)^{2}} \frac{1}{u_{2} v_{1}} .
$$

Therefore,

$$
\left[(a+2)+\frac{2}{a \rho(a+2)}\right]=-\frac{1}{(a+2)^{2} a \rho v_{2} u_{2}} .
$$

So $u_{2} v_{2}=-\left|u_{2} v_{2}\right|=-\sqrt{a} \rho$, and we get

$$
\rho=\frac{1}{\sqrt{a}(a+1)(a+2)} .
$$

Compare (2.64) with (2.62), we obtain

$$
a^{2}-1=\sqrt{a}(a+2) .
$$

Since $a>1$, we have

$$
\begin{aligned}
2\left(a^{3}+3 a^{2}-1\right)-a(a+2)^{2} & =a\left(a^{2}-1\right)+2\left(a^{2}-1\right)-3 a \\
& =\sqrt{a}(a+2)^{2}-3 a>(a+2)^{2}-3 a>0 .
\end{aligned}
$$

Thus, $f(w)>\frac{1}{2}$ by (2.63), a contradiction. Thus we proved Theorem 2.10. 
Remark 2.11. In view of the proof of Theorem 2.3 and 2.10, it is possible that for any $U(n)$-invariant Kähler metric on $\mathbb{C}^{n}$ the necessary condition for $k$-positive complex curvature operator $(2<k<n) \frac{k}{2(k-1)} A C-B^{2}>0$ is also sufficient.

\section{3. $U(n)$-invariant Kähler-Ricci flow on $\mathbb{C}^{n}$ with non-negative curvature}

\subsection{The $U(n)$-invariant Kähler-Ricci flow equation}

Throughout this section we will follow the notation from the Section 1. Pick any metric in $\mathcal{M}_{n}$, at the point $\left(z_{1}, 0, \ldots, 0\right)$ on $\mathbb{C}^{n}$, under the orthonormal frame $\left\{e_{1}=\frac{1}{\sqrt{h}} \partial_{z_{1}}, e_{2}=\frac{1}{\sqrt{f}} \partial_{z_{2}}, \ldots, e_{n}=\frac{1}{\sqrt{f}} \partial_{z_{n}}\right\}$, the non-zero components of the Ricci curvature are:

$$
\operatorname{Ric}\left(e_{1}, \bar{e}_{1}\right)=A+(n-1) B ; \operatorname{Ric}\left(e_{i}, \bar{e}_{i}\right)=B+\frac{n}{2} C ;
$$

where $2 \leq i \leq n$.

Assume that there exists a complete solution to the Ricci flow with $U(n)$-symmetry with initial metric in $\mathcal{M}_{n}$, we have:

$$
\left\{\begin{array}{l}
\frac{\partial h(z, t)}{\partial t}=-(A+(n-1) B) h(z, t), \\
\frac{\partial f(z, t)}{\partial t}=-\left(B+\frac{n}{2} C\right) f(z, t) .
\end{array}\right.
$$

Recall (2.5) and (2.6), we can simplify (3.2).

$$
\left\{\begin{array}{l}
\frac{\partial h(r, t)}{\partial t}=\frac{\partial}{\partial r}\left(\frac{r \frac{\partial h}{\partial r}}{h}\right)-(n-1)\left(\frac{h^{2}}{r f^{2}}-\frac{h(1-\xi)}{r f}\right) \\
\frac{\partial f(r, t)}{\partial t}=-\frac{\xi}{r}-\frac{n-1}{r}+(n-1) \frac{h}{r f} .
\end{array}\right.
$$

It is easy to see that the first equation in (3.3) can be derived from the second. To sum up, to get a complete Ricci flow with $U(n)$-symmetry it suffices to solve $f(r, t)$ on $[0,+\infty) \times[0, T)$ such that.

$$
\left\{\begin{array}{l}
\frac{\partial f(r, t)}{\partial t}=\frac{2 f_{r}+r f_{r r}}{f+r f_{r}}+(n-1) \frac{f_{r}}{f} \\
f(r, t)>0, \quad f+r f_{r}>0, \quad \int_{0}^{+\infty} \frac{\sqrt{f+r f_{r}}}{\sqrt{r}} d r=+\infty
\end{array}\right.
$$


When $n=1,(3.4)$ is reduced to the rotationally symmetric Ricci flow equation on $\mathbb{R}^{2}$. In general, the Ricci flow equation on $\mathbb{R}^{2}$ is related to a fast diffusion equation which was first studied by $\mathrm{Wu}$ [38] (see [20] and reference therein for further developments). It follows from the recent works [3, 15] that there always exists a complete solution to the Ricci flow with long time existence starting from a non-negatively curved $\mathbb{R}^{2}$ and its curvature becomes bounded instantaneously. In particular, Wu's result [38] implies that, assuming rotational symmetry, such a flow converges after modified by diffeomorphisms on $\mathbb{R}^{2}$. It was further proved in [38] that the limiting metric is Hamilton's cigar soliton if circumference at infinity of the initial metric is finite and it is the standard flat metric on $\mathbb{R}^{2}$ if the AVR is positive.

In higher dimensions, Fan [14] studied the uniqueness and convergence of $U(n)$-invariant Kähler-Ricci flow on $\mathbb{C}^{n}$ with positive bisectional curvature. However, his result assumes upper bounds on curvatures and relies on the short time existence theorem of Shi [34] and some earlier convergence results of Chau and Tam [6].

\subsection{Non-negativity of curvature is preserved}

Theorem 3.1. Let $g(t) t \in[0, T]$ be a complete solution of the Kähler-Ricci flow on $\mathbb{C}^{n}$ with $U(n)$-symmetry, if the holomorphic bisectional curvature of the initial metric $g(0)$ is non-negative, so is that of $g(t)$ for any $t \in[0, T]$. Moreover, if $g(0)$ has positive holomorphic bisectional curvature somewhere, then $g(t)$ has positive holomorphic bisectional curvature on $\mathbb{C}^{n} \times(0, T]$.

Proof of Theorem 3.1. We only need to prove that the non-negativity of the bisectional curvature is preserved, since the strong maximum principle is a local result and its proof is standard (see [18] and p. 193-195 of [13]). Given any complete $U(n)$-invariant Kähler-Ricci flow solution $g(t)$ on $\mathbb{C}^{n}$, we have a time-dependent orthonormal moving frame $\left\{e_{1}(t)=\frac{1}{\sqrt{h(t)}} \partial_{z_{1}}, e_{2}(t)=\right.$ $\left.\frac{1}{\sqrt{f(t)}} \partial_{z_{2}}, \ldots, e_{n}(t)=\frac{1}{\sqrt{f(t)}} \partial_{z_{n}}\right\}$ at the point $z=\left(z_{1}, 0, \ldots, 0\right)$. Denote

$$
\begin{aligned}
& A(z, t)=R m_{g(t)}\left(e_{1}(t), \bar{e}_{1}(t), e_{1}(t), \bar{e}_{1}(t)\right), \\
& B(z, t)=R m_{g(t)}\left(e_{1}(t), \bar{e}_{i}(t), e_{1}(t), \bar{e}_{i}(t)\right), \\
& C(z, t)=R m_{g(t)}\left(e_{i}(t), \bar{e}_{i}(t), e_{i}(t), \bar{e}_{i}(t)\right),
\end{aligned}
$$


where $2 \leq \mathrm{i} \leq n$. We have the evolution equation for bisectional curvature tensor

$$
\left\{\begin{array}{l}
\left(\frac{\partial}{\partial t}-\Delta\right) A=A^{2}+2(n-1) B^{2} \\
\left(\frac{\partial}{\partial t}-\Delta\right) B=-B^{2}+A B+\frac{n}{2} B C \\
\left(\frac{\partial}{\partial t}-\Delta\right) C=\frac{n}{2} C^{2}+2 B^{2}
\end{array}\right.
$$

Based on the following lemma, it suffices to show that $A(t) \geq 0$ is preserved along $g(t)$.

Lemma 3.2 Theorem 2, p. 525 in [37], see also Proposition 3.1 in [39]. For any complete Kähler metric $\omega$ on $\mathbb{C}^{n}$ with $U(n)$-symmetry, the bisectional curvature of $\omega$ is non-negative if and only if $A \geq 0$ everywhere.

Chen [10] proved that the non-negativity of the scalar curvature is always preserved along any complete solution to the Ricci flow (without assuming upper bounds on curvatures). The method is to apply the maximum principle for $u=\varphi\left(\frac{d_{t}\left(x, x_{0}\right)}{a r_{0}}\right) R(x, t)$ where $\varphi$ is a suitable cut-off function and $a>0$ is a sufficiently large constant. Recall that the scalar curvatures evolves by $\left(\frac{\partial}{\partial t}-\Delta\right) R=2 \mid$ Ric $\left.\right|^{2} \geq \frac{2}{n} R^{2}$, which is very similar to the evolution equation of $A$ in (3.6). Therefore, we can prove that $A \geq 0$ is preserved by the same method in [10].

Recall that Theorem 2.3 gives a characterization of non-negativity of various curvatures via $A, B$, and $C$. In particular, any complete $U(n)$-invariant Kähler metric with non-negative bisectional curvature has non-negative sectional curvature (non-negative complex curvature operator) if and only if $D=A C-B^{2} \geq 0\left(D_{n}=\frac{n}{2(n-1)} A C-B^{2} \geq 0\right)$.

Theorem 3.3. Let $g(t)$ be a complete solution of the Kähler-Ricci flow on $\mathbb{C}^{n}$ with $U(n)$-symmetry for $t \in[0, T]$. If the sectional curvature (complex curvature operator) of the initial metric $g(0)$ is non-negative, so is that of $g(t)$ for any $t \in(0, T]$. Moreover, if $g(0)$ has sectional curvature (complex curvature operator) positive somewhere, then $g(t)$ has positive sectional curvature (complex curvature operator) on $\mathbb{C}^{n} \times(0, T]$.

Proof of Theorem 3.3. First we will prove that the non-negativity of sectional curvature is preserved. Suppose there is a point $\left(z_{0}, t_{0}\right)$, where $0<t_{0} \leq$ 
$T$, where the sectional curvature is negative along some real 2-plane, then $D\left(z_{0}, t_{0}\right)=A C-B^{2}<0$. By picking $r_{0}>0$ small enough, we may assume that $\operatorname{Ric}(z, t) \leq \frac{n-1}{r_{0}^{2}}$ for any $z \in B_{t_{0}}\left(z_{0}, r_{0}\right)$, where $B_{t_{0}}\left(z_{0}, r_{0}\right)$ is with respect to $g\left(t_{0}\right)$.

$$
\begin{aligned}
\left(\frac{\partial}{\partial t}-\Delta\right)\left(A C-B^{2}\right) \\
=\left[\left(\frac{\partial}{\partial t}-\Delta\right) A\right] C+\left[\left(\frac{\partial}{\partial t}-\Delta\right) C\right] A \\
\quad-2 B\left[\left(\frac{\partial}{\partial t}-\Delta\right) B\right]-2 \nabla A \cdot \nabla C+2|\nabla B|^{2} \\
=A^{2} C+(n-2) B^{2} C+\frac{n}{2} C^{2} A+2 B^{3}-2 \nabla A \cdot \nabla C+2|\nabla B|^{2} .
\end{aligned}
$$

Let $\varphi$ is a fixed smooth cut-off non-increasing function such that $\varphi=1$ on $(-\infty, 1]$ and $\varphi=0$ on $[2,+\infty)$. Moreover,

$$
-2<\varphi^{\prime} \leq 0, \quad\left|\varphi^{\prime \prime}\right|+\frac{\left(\varphi^{\prime}\right)^{2}}{\varphi} \leq 32 .
$$

Define $u(z, t) \doteqdot \varphi\left(\frac{d_{t}\left(z, z_{0}\right)}{a r_{0}}\right) D(z, t)$, where $a>0$ will be a sufficiently large number.

$$
\begin{aligned}
\left(\frac{\partial}{\partial t}-\Delta\right) u= & \varphi^{\prime} \frac{1}{a r_{0}}\left[\left(\frac{\partial}{\partial t}-\Delta\right) d_{t}\right] D+\varphi\left[\left(\frac{\partial}{\partial t}-\Delta\right) D\right] \\
& -2 \nabla \varphi \cdot \nabla D-\varphi^{\prime \prime} \frac{D}{\left(a r_{0}\right)^{2}}
\end{aligned}
$$

Denote $u_{\min }(t)=\min _{z \in \mathbb{C}^{n}} u(z, t)$, so $u_{\min }\left(t_{0}\right) \leq u\left(z_{0}, t_{0}\right)<0$. Assume that there exists $\left(z_{1}, t_{1}\right)$ such that $u\left(z_{1}, t_{1}\right)=\min _{t \in[0, T]} u_{\min }(t)<0$. Now we compute the right-hand side of $(3.9)$ at the space-time point $\left(z_{1}, t_{1}\right)$. For simplicity, let us call it $Q\left(z_{1}, t_{1}\right)$.

First of all, Lemma 8.3 from Perelman [29] implies:

$$
\left(\frac{\partial}{\partial t}-\Delta\right) d_{t_{1}}\left(z, z_{0}\right) \geq-\frac{5(n-1)}{3 r_{0}}
$$

whenever $d_{t_{1}}\left(z, z_{0}\right)>r_{0}$.

The definition of $\left(z_{1}, t_{1}\right)$ implies $\nabla u\left(z_{1}, t_{1}\right)=0$. Therefore, $\nabla D=-\frac{\nabla \varphi}{\varphi} D$ and $\nabla A=\frac{1}{C}(\nabla D+2 B \nabla B-A \nabla C)$. 
It follows from (2.11), (2.12), and (2.13) and a straightforward calculation that

$$
\nabla_{s} B=\frac{2 x}{v}(A-2 B), \quad \nabla_{s} C=\frac{2 x}{v}(2 B-C) .
$$

$$
\begin{aligned}
Q\left(x_{1}, t_{1}\right) \geq & \varphi\left\{A^{2} C+(n-2) B^{2} C+\frac{n}{2} C^{2} A+2 B^{3}-2 \nabla A \cdot \nabla C+2|\nabla B|^{2}\right\} \\
& -\frac{10(n-1) \varphi^{\prime}}{3 a r_{0}^{2}} D+\frac{2\left(\varphi^{\prime}\right)^{2}}{\left(a r_{0}\right)^{2}} D-\frac{\varphi^{\prime \prime} D}{\left(a r_{0}\right)^{2}} \\
= & \varphi\left[A^{2} C+(n-2) B^{2} C+\frac{n}{2} C^{2} A+2 B^{3}\right] \\
& +\varphi\left[-\frac{2}{C} \nabla D \cdot \nabla C-\frac{4 B}{C} \nabla B \cdot \nabla C+\frac{2 A}{C}|\nabla C|^{2}+2|\nabla B|^{2}\right] \\
& -\frac{10(n-1) \varphi^{\prime}}{3 a r_{0}^{2}} D+\frac{2\left(\varphi^{\prime}\right)^{2}}{\left(a r_{0}\right)^{2}} D-\frac{\varphi^{\prime \prime} D}{\left(a r_{0}\right)^{2}} \\
\geq & \varphi\left[A^{2} C+(n-2) B^{2} C+\frac{n}{2} C^{2} A+2 B^{3}\right] \\
& +\varphi \frac{4 x^{2}}{v^{2}} \frac{2}{C}\left[A^{2} C+A C^{2}+8 B^{3}-6 A B C\right] \\
& -\frac{\varphi \prime}{\varphi} \frac{1}{a r_{0}} \frac{2 x}{C v}|2 B-C| D-\frac{10(n-1) \varphi^{\prime}}{3 a r_{0}^{2}} D+\frac{2\left(\varphi^{\prime}\right)^{2}}{\left(a r_{0}\right)^{2}} D-\frac{\varphi^{\prime \prime} D}{\left(a r_{0}\right)^{2}} .
\end{aligned}
$$

Claim 3.4. At the point $\left(z_{1}, t_{1}\right)$

$$
\begin{gathered}
A^{2} C+(n-2) B^{2} C+\frac{n}{2} C^{2} A+2 B^{3} \geq\left[B^{2}-A C\right]^{\frac{3}{2}}=|D|^{\frac{3}{2}} \\
A^{2} C+A C^{2}+8 B^{3}-6 A B C \geq 0 .
\end{gathered}
$$

Proof of Claim 3.4. Note that $B^{2}>A C$ at the point $\left(z_{1}, t_{1}\right),(3.13)$ can be verified by a straightforward calculation. Equation (3.14) simply follows from the the arithmetic and geometric mean inequality.

It follows from (3.12) that

$$
\left.\frac{d^{-} u_{\min }(t)}{d t}\right|_{t=t_{1}} \geq|u|^{\frac{3}{2}}+\left[-\frac{\varphi^{\prime}}{a r_{0}} C_{1}-\frac{\varphi^{\prime}}{a r_{0}^{2}} C_{2}+\frac{\left(\varphi^{\prime}\right)^{2} C_{3}}{\varphi\left(a r_{0}\right)^{2}}+\frac{\left|\varphi^{\prime \prime}\right|}{\left(a r_{0}\right)^{2}}\right] u
$$

where $C_{1}, C_{2}$ and $C_{3}$ are all constants depending only on the $g(t)$ restricted to a compact subset $\mathbb{C}^{n} \times[0, T]$. 
On the other hand, the choice of the point $\left(z_{1}, x_{1}\right)$ implies $\frac{d^{-} u_{\min }(t)}{d t} \leq 0$. We conclude that $\sqrt{\left|u\left(x_{1}, t_{1}\right)\right|} \leq \frac{C_{5}}{a r_{0}}+\frac{C_{6}}{\left(a r_{0}\right)^{2}}$. Therefore, we have

$$
D\left(x_{0}, t_{0}\right) \geq u\left(x_{1}, t_{1}\right) \geq-\left[\frac{C_{5}}{a r_{0}}+\frac{C_{6}}{\left(a r_{0}\right)^{2}}\right]^{2}
$$

Now let $a$ goes to infinity, we get $D\left(z_{0}, t_{0}\right) \geq 0$, which contradicts to the choice of $\left(z_{0}, t_{0}\right)$. Therefore, the first part of Theorem 3.3 is proved.

It remains to show that the condition $D_{n}=\frac{n}{2(n-1)} A C-B^{2} \geq 0$ is preserved. Let us write $D_{\lambda}=\lambda A C-B^{2}$. We will next prove that, for any $\lambda \in$ $\left[\frac{1}{2}, 1\right]$, the condition $D_{\lambda} \geq 0$ is preserved under the $U(n)$-invariant KählerRicci flow. We show it by using a successive approximation on $\lambda$.

Follow the proof above for the preservation of the condition $D_{1} \geq 0$, a similar computation shows:

$$
\begin{aligned}
& \left(\frac{\partial}{\partial t}-\Delta\right)\left[\varphi\left(\frac{d_{t}\left(z, z_{0}\right)}{a r_{0}}\right) D_{\lambda}(z, t)\right] \\
& \geq \varphi\left[\lambda A^{2} C+(2 \lambda(n-1)-n) B^{2} C+\frac{\lambda n}{2} C^{2} A+2 B^{3}-2(1-\lambda) A B^{2}\right] \\
& \quad+\varphi \frac{4 x^{2}}{v^{2}} \frac{2}{C}\left[\lambda A^{2} C+A C^{2}+8 B^{3}-(2+4 \lambda) A B C-(4-4 \lambda) A B^{2}\right] \\
& \quad-\frac{\varphi}{\varphi} \frac{1}{a r_{0}} \frac{2 x}{C v}|2 B-C| D_{\lambda}-\frac{10(n-1) \varphi^{\prime}}{3 a r_{0}^{2}} D_{\lambda}+\frac{2\left(\varphi^{\prime}\right)^{2}}{\left(a r_{0}\right)^{2}} D_{\lambda}-\frac{\varphi^{\prime \prime} D_{\lambda}}{\left(a r_{0}\right)^{2}}
\end{aligned}
$$

Denote

$$
\begin{aligned}
& I_{1}(\lambda)=\lambda A^{2} C+(2 \lambda(n-1)-n) B^{2} C+\frac{\lambda n}{2} C^{2} A+2 B^{3}-2(1-\lambda) A B^{2} \\
& I_{2}(\lambda)=A^{2} C+A C^{2}+8 B^{3}-(2+4 \lambda) A B C-(4-4 \lambda) A B^{2} .
\end{aligned}
$$

The key observation is that:

Lemma 3.5. There exists a decreasing sequence $\left\{\lambda_{k}\right\}$ with the following property:

(1) $\lambda_{0}=1$ and $\frac{1}{2}<\lambda_{k}<1$ for any $k>1$.

(2) Assume $\lambda_{k+1} A C<B^{2} \leq \lambda_{k} A C$ at the point $\left(z_{1}, t_{1}\right)$, then:

$$
\begin{gathered}
I_{1}\left(\lambda_{k+1}\right) \geq\left[B^{2}-\lambda_{k+1} A C\right]^{\frac{3}{2}}=\left|D_{\lambda_{k+1}}\right|^{\frac{3}{2}}, \\
I_{2}\left(\lambda_{k+1}\right) \geq 0 .
\end{gathered}
$$


Proof of Lemma 3.5. Denote $K \doteqdot \frac{A C}{B^{2}}$, where $\frac{1}{\lambda_{k}} \leq K<\frac{1}{\lambda_{k+1}}$.

$$
\begin{aligned}
& I_{2}\left(\lambda_{k+1}\right) \\
& \quad=A^{2} C+A C^{2}+8 B^{3}-\left(2+4 \lambda_{k+1}\right) A B C-\left(4-4 \lambda_{k+1}\right) A B^{2} \\
& \quad=A B^{2}\left\{\lambda_{k+1} K^{2}\left(\frac{B}{A}\right)^{2}+\left[8-\left(2+4 \lambda_{k+1}\right) K\right] \frac{B}{A}+K-4\left(1-\lambda_{k+1}\right)\right\} .
\end{aligned}
$$

To ensure that $I_{2}\left(\lambda_{k+1}\right)$ is non-negative, it suffices to have $\frac{1}{\lambda_{k}} \geq 4(1-$ $\left.\lambda_{k+1}\right)$ and $\lambda_{k+1} \geq \frac{1}{2}$. This motivates us to define the sequence $\left\{\lambda_{k}\right\}$ recursively by $\lambda_{k+1}=1-\frac{1}{4 \lambda_{k}}$ with $\lambda_{1}=1$. In fact, it is easy to check that $\lambda_{k}=$ $\frac{k+1}{2 k}$.

$$
\begin{aligned}
I_{1}\left(\lambda_{k+1}\right)= & \lambda_{k+1} A^{2} C+\left(2 \lambda_{k+1}(n-1)-n\right) B^{2} C \\
& +\frac{\lambda_{k+1} n}{2} C^{2} A+2 B^{3}-2\left(1-\lambda_{k+1}\right) A B^{2} \\
= & A B^{2}\left\{\left[2 \lambda_{k+1}(n-1)-n+\frac{\lambda n}{2} K\right] K\left(\frac{B}{A}\right)^{2}\right. \\
& \left.+2 \frac{B}{A}+\lambda_{k+1} K-2\left(1-\lambda_{k+1}\right)\right\}
\end{aligned}
$$

and

$$
\left[B^{2}-\lambda_{k+1} A C\right]^{\frac{3}{2}}=\left(1-\lambda_{k+1} K\right) B^{3}
$$

It follows from (3.21) and (3.22) that $\frac{\lambda_{k+1}}{\lambda_{k}} \geq 2-2 \lambda_{k+1} \geq 0$ and $2 \lambda_{k+1}$ $(n-1)-n+\frac{n}{2} \frac{\lambda_{k+1}}{\lambda_{k}} \geq 0$ will suffice to prove (3.18). It is straightforward to check that those two inequalities are satisfies if we let $\lambda_{k}=\frac{k+1}{2 k}$.

From Lemma 3.5 and the proof of the preservation of the condition $D_{1} \geq$ 0 , we can argue inductively to show that $D_{\lambda} \geq 0$ is preserved for any $\frac{1}{2}<$ $\lambda \leq 1$. Thus, we have proved that $D_{n}=\frac{n}{2(n-1)} A C-B^{2}$ is preserved along the Ricci flow with $U(n)$-symmetry. To sum up, we have shown that any non-negative curvature condition which lies between non-negative sectional curvature and non-negative complex curvature operator is preserved along any complete solution to Kähler-Ricci flow on $\mathbb{C}^{n}$ with $U(n)$-symmetry. 


\subsection{The asymptotic volume ratio is preserved}

Recall that the AVR of a complete non-compact Riemannian manifold $\left(N^{n}, h\right)$ is defined by

$$
\operatorname{AVR}(h) \doteqdot \lim _{s \rightarrow \infty} \frac{\operatorname{Vol} B(p, s)}{\omega_{n} s^{n}},
$$

provided that the limit exists. (E.g., when $\left(N^{n}, h\right)$ has non-negative Ricci curvature.) Here, $B(p, s)$ denotes the geodesic ball in $N$ with radius $r$ and centered at $p$, and $\omega_{n}$ is the volume of the unit Euclidean $n$-ball.

Hamilton [19] proved that AVR is constant on any complete Ricci flow with bounded non-negative Ricci curvature if the Riemannian curvature decays pointwisely to zero along infinity on each time slice. In the case of Kähler-Ricci flow, Shi [33] proved that the maximal volume growth is preserved for a complete Kähler-Ricci flow with bounded bisectional curvature in the space time and with the scalar curvature of the initial metric having average quadratic decay. Shi's result was improved by weaker assumptions on the scalar curvature decay in more recent works; see Chen and Zhu [12], Chen et al. [11] and $\mathrm{Ni}$ and Tam [27], for example. There are also similar results for the Ricci flow on Riemannian manifolds with non-negative bounded curvature operator (Yokota [42] and Schulze and Simon [31]). In particular, Ni and Tam [27] proved that the order of volume growth of geodesics balls in each time slice keeps constant on complete Kähler-Ricci flow with bounded non-negative bisectional curvature in space-time. Our main result in this subsection is to remove the upper bound of curvature with the help of $U(n)$-symmetry.

Theorem 3.6. Let $g(t), t \in[0, T]$, be a complete solution to the KählerRicci flow on $\mathbb{C}^{n}$ with $U(n)$-symmetry. Assume that the bisectional curvature of $g(t)$ is non-negative for any $t \in[0, T]$. Then for any $t \in[0, T]$

$$
\lim _{s \rightarrow+\infty} \frac{V_{t}\left(B_{t}(O, s)\right)}{V_{0}\left(B_{0}(O, s)\right)}=1
$$

where $B_{t}(O, s)$ denotes the geodesic ball of radius $s$ for the metric $g(t)$ centered at the origin. In particular, the AVR of $\left(\mathbb{C}^{n}, g(t)\right)$ is constant for any $t \in[0, T]$.

Recall that for any fixed metric $g_{0}$ in $\mathcal{M}_{n}$, at the point $z=\left(z_{1}\right.$, $0, \ldots, 0)$ on $\mathbb{C}^{n}$, under the orthonormal frame $\left\{e_{1}=\frac{1}{\sqrt{h}} \partial_{z_{1}}, e_{2}=\frac{1}{\sqrt{f}}\right.$ 
$\left.\partial_{z_{2}}, \ldots, e_{n}=\frac{1}{\sqrt{f}} \partial_{z_{n}}\right\}:$

$$
\operatorname{Ric}\left(e_{1}, \bar{e}_{1}\right)=A+(n-1) B
$$

and the distance between the origin and a point $z$ is given by

$$
s=\int_{0}^{r} \frac{\sqrt{h}}{2 \sqrt{r}} d r
$$

where $r=|z|^{2}$. The following lemma gives an integral bound for the Ricci curvature along the radial geodesic. It further implies a lower bound on the radial distance function along $U(n)$-invariant Kähler-Ricci flow.

Lemma 3.7. There exists a constant $C_{0}$ that only depends on $g_{0}$ restricted on the fixed coordinate ball such that

$$
\int_{0}^{s} \operatorname{Ric}\left(\frac{\partial}{\partial s}, \frac{\partial}{\partial s}\right) d s \leq C_{0}
$$

Proof of Lemma 3.7.

$$
\int_{0}^{s} \operatorname{Ric}\left(e_{1}, \bar{e}_{1}\right) d s=\int_{0}^{r} \frac{[A+(n-1) B] \sqrt{h}}{2 \sqrt{r}} d r
$$

Recall (2.11),(2.12), and (2.13) and let $a=\sqrt{h(1)}>0$.

$$
\begin{aligned}
\int_{1}^{r} \frac{A \sqrt{h}}{2 \sqrt{r}} d r & =\int_{a}^{x} \frac{F^{\prime} F^{\prime \prime}}{2 \tau\left[1+F^{\prime}(\tau)^{2}\right]^{\frac{3}{2}}} d \tau \\
& =\int_{a}^{x} \frac{1}{2 \tau}\left(\frac{-1}{\sqrt{1+F^{\prime}(\tau)^{2}}}\right) d \tau \\
& \leq \frac{1}{2 a}-\frac{1}{2 x \sqrt{1+F^{\prime}(x)^{2}}}-\int_{a}^{x} \frac{1}{2 \tau^{2} \sqrt{1+F^{\prime}(\tau)^{2}}} d \tau \\
& \leq \frac{1}{2 a}
\end{aligned}
$$


$(3.30)$

$$
\begin{aligned}
\int_{1}^{r} \frac{B \sqrt{h}}{2 \sqrt{r}} d r & =\int_{a}^{x} \frac{1}{v^{2}}\left(\tau^{2}-\frac{v}{\sqrt{1+F^{\prime}(\tau)^{2}}}\right) \sqrt{1+F^{\prime}(\tau)^{2}} d \tau \\
& \leq \frac{1}{2} \frac{a}{v(a)}+\frac{1}{2} \int_{a}^{x} \frac{1}{v}\left[-1+\frac{v}{\tau^{2} \sqrt{1+F^{\prime 2}}}-\frac{v}{\tau}\left(\frac{1}{\sqrt{1+F^{\prime 2}}}\right)^{\prime}\right] d \tau \\
& \leq \frac{1}{2} \frac{a}{v(a)}+\frac{1}{2}\left(\int_{a}^{x} \frac{2}{\tau^{2}} d \tau+\frac{1}{a}\right) \\
& \leq \frac{1}{2} \frac{a}{v(a)}+\frac{3}{2 a} .
\end{aligned}
$$

As in the work of Shi [34], we define

$$
F(x, t)=\log \left(\frac{\operatorname{det} g(x, t)}{\operatorname{det} g(x, 0)}\right) \text {. }
$$

and Let $\Delta_{t}$ and $d V_{t}$ denote the Laplacian operator and the volume element with respect to $g(t)$.

Lemma 3.8. Let $g(t), t \in[0, T]$, be a complete solution to the KählerRicci flow on $\mathbb{C}^{n}$ with $U(n)$-symmetry and non-negative bisectional curvature. Then for any fixed $t \in(0, T]$, there exists a constant $C_{0}$ which only depends on $n$ and $g(t)$ restricted to a compact set of $\mathbb{C}^{n} \times[0, t]$, such that:

$$
f_{B_{0}(O, s)}\left(1-\mathrm{e}^{F(x, t)}\right) d V_{0} \leq \frac{C_{0} t}{s^{2}}\left[\int_{0}^{10 s}\left[s f_{B_{0}(O, s)} R(x, 0) d V_{0}\right] d s+s\right] .
$$

Proof of Lemma 3.8. It will follows from a slight modification of the method in Theorem 2.2 on p. 132 of $\mathrm{Ni}$ and Tam [27]. In particular we need their estimate (3.38) whose proof is also included for the convenience of the reader. In [27] the result was stated under the assumption that $g(t)$ has bounded curvature along $[0, T]$; here we are able to remove the assumption on curvature bounds with aid of the $U(n)$-symmetry.

First we have the following inequality from Shi [34]:

$$
\Delta_{0} F(x, t) \leq R(x, 0)+\mathrm{e}^{F} \frac{\partial F(x, t)}{\partial t} .
$$


Let $G_{s}(x, y)$ be the positive Green's function on $B_{0}(O, s)$ with zero boundary value. Integrate $(3.33)$ over $B_{0}(O, s) \times[0, t]$, we get for $B_{0}=$ $B_{0}(O, s)$ that:

$$
\begin{aligned}
\int_{B_{0}} & G_{s}(O, x)\left(1-\mathrm{e}^{F(x, t)}\right) d V_{0} \\
\leq & t \int_{B_{0}} G_{s}(O, x) R(x, 0) d V_{0}-\int_{0}^{t} \int_{B_{0}} G_{s}(O, x) \Delta_{0} F(x, \tau) d V_{0} d \tau \\
= & t \int_{B_{0}} G_{s}(O, x) R(x, 0) d V_{0} \\
& +\int_{0}^{t}\left[F(O, \tau)+\int_{\partial B_{0}} F(x, \tau) \frac{\partial G_{s}(O, x)}{\partial s} d A_{0}\right] d \tau \\
\leq & t\left[\int_{B_{0}} G_{s}(O, x) R(x, 0) d V_{0}-\left.F(x, t)\right|_{\partial B_{0}}\right]
\end{aligned}
$$

where $d A_{0}$ is the area element for $\partial B_{0}$.

Note that in (3.34) we used the Green's formula and the facts that $F(x, t) \leq 0$ is non-increasing and $\int_{\partial B_{0}(O, s)} \frac{\partial G_{s}(O, x)}{\partial s} d A_{0}=-1$.

As in $[12,27,34]$, considering $\mathbb{C}^{n} \times \mathbb{C}^{2}$, we may assume that $\left(\mathbb{C}^{n}, g(0)\right)$ admits a minimal positive Green's function such that

$$
\alpha \frac{d^{2}(x, y)}{V(x, d(x, y))} \leq G(x, y) \leq \frac{1}{\alpha} \frac{d^{2}(x, y)}{V(x, d(x, y))}
$$

for some $\alpha>0$, which depends only on $n$.

Then we can apply the mean value inequality in [26] (see the proof of Theorem 1.1 and Theorem 2.1 on p. $345-348$ of [26]) to conclude that

$$
\begin{aligned}
& \int_{B_{0}(O, s)} G_{s}(O, x) R(x, 0) d V_{0} \\
& \quad \leq \int_{B_{0}(O, s)} G(O, x) R(x, 0) d V_{0} \\
& \quad \leq c(n, \alpha) \int_{0}^{2 s}\left[s f_{B_{0}(O, s)} R(x, 0) d V_{0}\right] d \tau, \\
& s^{2} \int_{B_{0}(O, s)}\left(1-\mathrm{e}^{F(x, t)}\right) d V_{0} \\
& \quad \leq c(n, \alpha) \int_{B_{0}(O, 5 s)} G_{s}(O, x)\left(1-\mathrm{e}^{F(x, t)}\right) d V_{0} .
\end{aligned}
$$


Now (3.34) becomes

$$
\begin{aligned}
& s^{2} f_{B_{0}(O, s)}\left(1-\mathrm{e}^{F(x, t)}\right) d V_{0} \\
& \quad \leq c(n) t\left[\int_{0}^{10 s}\left[s f_{B_{0}(O, s)} R(x, t) d V_{0}\right] d s-F(x, t)\right] .
\end{aligned}
$$

We remark that similar estimates as (3.38) have been used in $[11,12,33]$. The proof of Lemma 3.8 will be done if we can prove

$$
-\left.F(x, t)\right|_{\partial B_{0}(0, s)} \leq C_{0} s
$$

for a constant $C_{0}$ which depends only on $g(t)$ restricted to a compact set in $\mathbb{C}^{n}$.

Since $\left(\mathbb{C}^{n}, g(t)\right)$ has $U(n)$-symmetry and positive curvature, we have for any $r \geq 1$

$$
s=\int_{0}^{r} \frac{1}{2} \sqrt{\frac{h(r)}{r}} d t \geq \frac{\sqrt{h(1)}}{2} \log r .
$$

Recall the definition of $F$ in (3.31),

$$
F=\log h(x, t)+(n-1) \log f(x, t)-\log h(x, 0)-(n-1) \log f(x, 0),
$$

$$
-r F_{r} \leq \frac{-r h_{r}(x, t)}{h(x, t)}+(n-1) \frac{-r f_{r}(x, t)}{f(x, t)} \leq \xi(+\infty, t)+\frac{f(r, t)-h(r, t)}{f(r, t)} \leq n .
$$

Now from (3.42) and (3.40) we have

$$
-F(r, t) \leq n \log r-F(1, t) \leq C_{0} s
$$

which completes the proof of Lemma 3.8.

Proof of Theorem 3.6. Theorem 3.6 can be proved by arguing similarly as the proof of Theorem 2.2 in [27]. First note that Lemma 3.7 and the volume comparison theorem will imply that the AVR of $g(t)$ is non-increasing on $[0, T]$. 
As in $[12,27]$, we have

$$
V_{t}\left(B_{t}(O, s)\right) \geq \int_{B_{0}(O, s)} d V_{t} \geq V_{0}\left(B_{t}(O, s)\right)-\int_{B_{0}(O, s)}\left(1-\mathrm{e}^{F(x, t)}\right) d V_{0} .
$$

Recall that we have decay estimates of the average scalar curvature for $U(n)$-invariant Kähler metrics on $\mathbb{C}^{n}$ with non-negative curvature, see Theorem 7 in [37] or Proposition 3.3 in [39]). We conclude from Lemma 3.8 that the AVR of $g(t)$ is non-decreasing on $[0, T]$. Hence it must be constant for any $t \in[0, T]$. In fact, the proof shows that the precise order of volume growth of $\left(\mathbb{C}^{n}, g(t)\right)$ is constant on $[0, T]$, i.e., (3.24) must hold.

\section{Discussions on the existence of $U(n)$-invariant Kähler-Ricci flow}

\subsection{Theorem of Cabezas-Rivas and Wilking}

Let us recall the following result by Cabezas-Rivas and Wilking. [3]:

Theorem 4.1 Cabezas-Rivas and Wilking [3]. Let $\left(M^{n}, g\right)$ be an open manifold with non-negative complex sectional curvature. Then there exists a constant $T>0$ which depends on $n$ and $g$ such that there exists a complete solution of Ricci flow $g(t)$ with non-negative complex sectional curvature starting from $g$ on $[0, T]$. In addition, if we assume

$$
\inf \left\{\operatorname{Vol}_{g} B_{g}(p, 1): p \in M\right\}=v_{0}>0,
$$

then $T$ can be chosen as $T\left(n, v_{0}\right)$ and the scalar curvature of $(M, g(t))$ is bounded by $\frac{c\left(n, v_{0}\right)}{t}$ on $\left(0, T\left(n, v_{0}\right)\right]$.

\subsection{Ricci flow on double covers with rotational symmetry}

Let us consider $\left(\mathbb{C}^{n}, g_{0}\right)$, where $g_{0}$ is a complete $U(n)$-invariant Kähler metric with non-negative complex sectional curvature. In fact, by Theorem 1.5 we only need to assume that $g_{0}$ has non-negative sectional curvature. Let $B(O, i)$ denote the geodesic ball with radius $i$ centered at the origin. The double cover $D_{i}$ is a closed manifold obtained by gluing two copies of $B(O, i)$ after identifying the boundary and perturbing the inner region nearby. To be more precise, define a smooth function $\phi_{i}:(-\infty, i) \rightarrow \mathbb{R}$ with $\phi_{i}=0$ on $(-\infty, i-\epsilon]$ and $\phi_{i}(i)=1, \phi_{i}^{\prime}, \phi_{i}^{\prime \prime}>0$ on $(i-\epsilon, i)$, and $\phi_{i}$ is 
left continuous at $i$ and left derivatives of its inverse vanishes at 1 . Then define $F_{i}: D_{i} \rightarrow \mathbb{C}^{n} \times \mathbb{R}$ by $F_{i}(z)=\left(z, \phi_{i}(s(z))\right)$ on one copy of $B(O, i)$ and $F_{i}(z)=\left(z, 2-\phi_{i}(s(z))\right)$ on the other. It can be checked that this will realize $D_{i}$ as a closed smooth hypersurface in $\mathbb{C}^{n} \times \mathbb{R}$. The induced metric of $D_{i}$ from the product metric of $\mathbb{C}^{n} \times \mathbb{R}$, denoted by $g_{i}$, has non-negative complex sectional curvature. It follows from Proposition 4.1 in $[3]$ that $\left(D_{i}, g_{i}, O\right)$ converges to $\left(\mathbb{C}^{n}, g_{0}, O\right)$ smoothly in the sense of Cheeger-Gromove convergence (see [3] p. 7-8 for the details of the results mentioned above).

The general Theorem 4.1 is proved by Cabezas-Rivas and Wilking by establishing some delicate curvature estimates on the closed Ricci flow evolved from those double covers. In this section, under the extra assumption of the rotational symmetry of $\left(\mathbb{C}^{n}, g_{0}\right)$, we will compute the curvatures of $\left(D_{i}, g_{i}\right)$ and its evolution under the Ricci flow more explicitly.

Fix a point $p=\left(x_{1}, \ldots, x_{2 n}\right)$ on $\left(D_{i}, g_{i}\right)$ where $x_{k}=0$ for $2 \leq k \leq 2 n$. Define $r=\sum_{k=1}^{2 n}\left|x_{k}\right|^{2}$ and the function $s(r)=\int_{0}^{r} \frac{\sqrt{h(r)}}{2 \sqrt{r}} d r$. Note that $s(r)$ is the distance function only inside the ball $B(O, i-\epsilon)$. Under the local coordinates $\left\{x_{1}, \ldots, x_{2 n}\right\}$, the metric $\left(D_{i}, g_{i}\right)$ has components:

$$
\begin{aligned}
g_{i}\left(\frac{\partial}{\partial x_{1}}, \frac{\partial}{\partial x_{1}}\right) & =h\left(2+\left(\phi_{i}^{\prime}(s)\right)^{2}\right), \quad g_{i}\left(\frac{\partial}{\partial x_{n+1}}, \frac{\partial}{\partial x_{n+1}}\right)=2 h . \\
g_{i}\left(\frac{\partial}{\partial x_{k}}, \frac{\partial}{\partial x_{k}}\right) & =g_{i}\left(\frac{\partial}{\partial x_{n+k}}, \frac{\partial}{\partial x_{n+k}}\right)=2 f . \quad(2 \leq k \leq n) \\
g_{i}\left(\frac{\partial}{\partial x_{p}}, \frac{\partial}{\partial x_{q}}\right) & =0(p \neq q) .
\end{aligned}
$$

At the same point, we calculate the non-zero components of the second fundamental form of $\left(D_{i}, g_{i}\right)$ with respect to $\mathbb{C}^{n} \times \mathbb{R}$. Note that we abuse the notations again by denoting $\frac{\partial}{\partial x_{k}}$ for $d F_{i}\left(\frac{\partial}{\partial x_{k}}\right)$.

$$
\begin{aligned}
& \Pi\left(\frac{\partial}{\partial x_{1}}, \frac{\partial}{\partial x_{1}}\right)=\frac{\sqrt{2} \phi_{i}^{\prime \prime}(s)}{\sqrt{2+\left(\phi_{i}^{\prime}\right)^{2}}} h \\
& \Pi\left(\frac{\partial}{\partial x_{n+1}}, \frac{\partial}{\partial x_{n+1}}\right)=\frac{\sqrt{2} \phi_{i}^{\prime}(s)}{\sqrt{2+\left(\phi_{i}^{\prime}\right)^{2}}}\left(\sqrt{\frac{h}{r}}-\sqrt{\frac{r}{h}} h_{r}\right), \\
& \Pi\left(\frac{\partial}{\partial x_{k}}, \frac{\partial}{\partial x_{k}}\right)=\Pi\left(\frac{\partial}{\partial x_{n+k}}, \frac{\partial}{\partial x_{n+k}}\right)=\frac{\sqrt{2} \phi_{i}^{\prime}(s)}{\sqrt{2+\left(\phi_{i}^{\prime}\right)^{2}}} \sqrt{\frac{h}{r}} .
\end{aligned}
$$

The Gauss equation gives the curvature of $\left(D_{i}, g_{i}\right)$ under the complexified local coordinates $z_{k}=x_{k}+\sqrt{-1} x_{n+k}$. We should caution here that 
these $z_{k}$ are only holomorphic inside the ball $B(O, i-\epsilon) \subset D_{i}$. We list all the non-zero curvature components below.

$$
\begin{aligned}
& R_{1 \overline{1} 1 \overline{1}}=h^{2} A+\Pi_{1 \overline{1}}^{2}-\left|\Pi_{11}\right|^{2}, \quad R_{1 \overline{1} k \bar{k}}=h f B \\
& R_{k \bar{k} k \bar{k}}=f^{2} C+\Pi_{k \bar{k}}^{2}, \quad R_{k \bar{k} l \bar{l}}=\frac{1}{2} f^{2} C \\
& R_{\bar{k} \overline{1} 1 k}=\Pi_{k \bar{k}} \Pi_{1 \overline{1}}, \quad R_{\bar{k} 11 k}=\Pi_{k \bar{k}} \Pi_{11} .
\end{aligned}
$$

Under the orthonormal frame $e_{1}=\frac{1}{\sqrt{h\left(2+\left(\phi_{i}^{\prime}(s)\right)^{2}\right)}} \partial_{x_{1}}, e_{2}=\frac{1}{\sqrt{2 f}} \partial_{x_{2}}, \ldots$, $e_{n+1}=\frac{1}{\sqrt{2 h}} \partial_{z_{n+1}}, \ldots, e_{n+2}=\frac{1}{\sqrt{2 f}} \partial_{x_{n+2}}, \ldots, e_{2 n}=\frac{1}{\sqrt{2 f}} \partial_{x_{2 n}}$ and its corresponding complexification $\left(\omega_{1}, \ldots, \omega_{n}\right)$ with $\omega_{k}=\frac{1}{\sqrt{2}}\left(e_{k}-\sqrt{-1} e_{n+k}\right)$, it is easy to see that

$$
g_{i}\left(\omega_{k}, \bar{\omega}_{l}\right)=\delta_{k l}, \quad g_{i}\left(\omega_{k}, \omega_{l}\right)=0 .
$$

It is easy to derive the formula for the curvature components of $\left(D_{i}, g_{i}\right)$ under the orthonormal frame $\omega_{k}$. It has similar type of non-zero components as that under the coordinates $z_{k}$ as in (4.4).

To simplify the computation of the curvature evolution of the Ricci flow on the double cover, we use the Uhlenbeck trick to evolve the above complexified orthonormal frame $\omega_{k}$ to get a time-dependent orthonormal frame $\left(\omega_{1}(t), \ldots, \omega_{n}(t)\right)$ with the property that (4.5) holds for any $t$.

Lemma 4.2. The complete Ricci flow $\left(\mathbb{C}^{n}, g(t)\right)$ constructed in Theorem 4.1 is a Kähler-Ricci flow on $\mathbb{C}^{n}$ with $U(n)$-symmetry if $R_{A B \gamma \delta}(g(t))=0$ everywhere under the time-dependent orthonormal frame $\left(\omega_{1}(t), \ldots, \omega_{n}(t)\right)$. Here $A, B$ are any indices either barred or unbarred while $\gamma$ and $\delta$ are unbarred from 1 to $n$.

Proof of Lemma 4.2. The fact that $g(t)$ being Kähler is proved by Shi (See p. 138-142 [34]). Here the $U(n)$-symmetry follows from the rotational symmetry of $\left(\mathbb{C}^{n}, g_{0}\right)$ and $\left(D_{i}, g_{i}(t)\right)$.

\subsection{Conditions that ensure the existence of Kähler-Ricci flow}

From now on we focus our discussion in the complex dimension $n=2$, we emphasize that it is purely for the sake of convenience and all results mentioned below can be generalized to higher dimensions.

The following two results study the curvature evolution of the closed Ricci flow $\left(D_{i}, g_{i}(t)\right)$ under the time-dependent orthnormal frame 
$\left(\omega_{1}(t), \omega_{2}(t)\right)$. Note that the only non-vanishing curvature components of the initial metric $\left(D_{i}, g_{i}(0)\right)$ are $R_{1 \overline{1} 1 \overline{1}}, R_{1 \overline{1} 2 \overline{2}}, R_{2 \overline{2} 2 \overline{2}}, R_{\overline{2} 112}$, and $R_{\overline{2} \overline{1} 12}$.

Lemma 4.3. The curvatures of $\left(D_{i}, g_{i}(t)\right)$ satisfy:

$$
R_{1 \overline{1} 12}=R_{1 \overline{1} 2 \overline{1}}=R_{2 \overline{2} 1 \overline{2}}=R_{2 \overline{2} 21}=0 \text {. }
$$

Proof of Lemma 4.3. We have the following curvature evolution equations via the Uhlenbeck trick.

$$
\begin{aligned}
\left(\frac{\partial}{\partial t}-\Delta\right) R_{1 \overline{1} 21} & =-R_{1 \overline{1} E G} R_{\bar{E} \bar{G} 21}+2 R_{E 1 G 1} R_{\bar{E} \overline{1} \bar{G} 2}-R_{E 1 G 2} R_{\bar{E} \overline{1} \bar{G} 1} \\
& =Q_{1}\left(R_{1 \overline{1} 21}, R_{1 \overline{1} 1 \overline{2}}, R_{1 \overline{2} 21}\right)
\end{aligned}
$$

Similarly:

$$
\begin{aligned}
& \left(\frac{\partial}{\partial t}-\Delta\right) R_{1 \overline{1} 1 \overline{2}}=Q_{2}\left(R_{1 \overline{1} 21}, R_{1 \overline{1} 1 \overline{2}}, R_{2 \overline{2} 1 \overline{2}}, R_{2 \overline{2} 12}\right) \\
& \left(\frac{\partial}{\partial t}-\Delta\right) R_{2 \overline{2} 12}=Q_{3}\left(R_{1 \overline{1} 21}, R_{1 \overline{1} 1 \overline{2}}, R_{2 \overline{2} 1 \overline{2}}, R_{2 \overline{2} 12}\right) \\
& \left(\frac{\partial}{\partial t}-\Delta\right) R_{2 \overline{2} 2 \overline{1}}=Q_{4}\left(R_{1 \overline{1} 21}, R_{1 \overline{1} 1 \overline{2}}, R_{2 \overline{2} 1 \overline{2}}, R_{2 \overline{2} 12}\right) .
\end{aligned}
$$

Note that $R_{1 \overline{1} 12}=R_{1 \overline{1} 2 \overline{1}}=R_{2 \overline{2} 1 \overline{2}}=R_{2 \overline{2} 21}=0$ holds at $t=0$, so the lemma follows from the maximum principle for solutions to parabolic equations on closed manifolds.

Similarly, we can prove the following lemma.

Lemma 4.4. The curvatures of $\left(D_{i}, g_{i}(t)\right)$ satisfy:

$$
R_{1221}=R_{2 \overline{1} 21}=R_{2 \overline{1} 2 \overline{1}}=0
$$

After passing the time-dependent orthnormal frame $\left(\omega_{1}(t), \omega_{2}(t)\right)$ on $\left(D_{i}, g_{i}(t)\right)$ to the limit Ricci flow $\left(\mathbb{C}^{2}, g(t)\right)$, we have a time-dependent orthnormal frame on $\left(\mathbb{C}^{2}, g(t)\right)$. Let $\left(\omega_{1}(t), \omega_{2}(t)\right)$ still denote the frame $\left(\mathbb{C}^{2}, g(t)\right)$ for simplicity, the above lemmas show that all curvatures components of the type $R_{A B \gamma \delta}(g(t))$ of $\left(\mathbb{C}^{2}, g(t)\right)$ vanish except $R_{\overline{2} 112}$ and $R_{\overline{2} \overline{1} 12}$.

Theorem 4.5. Assume $\left(\mathbb{C}^{2}, g_{0}\right)$ is a complete $U(2)$-invariant Kähler manifold with non-negative sectional curvature. Moreover, assume that the condition (4.1) holds.(e.g., (4.1) is satisfied when $\left(\mathbb{C}^{2}, g_{0}\right)$ has Euclidean 
volume growth.) Then Cabezas-Rivas and Wilking Ricci flow in Theorem 4.1 satisfies either

$$
R_{\overline{2} 112}=R_{\overline{2} \overline{1} 12}=0
$$

everywhere on $\mathbb{C}^{2} \times\left[0, T\left(2, v_{0}\right)\right]$ or $P(x, t)>0$ everywhere and

$$
\liminf _{r_{0} \rightarrow+\infty, t_{0} \rightarrow 0} \frac{\inf _{B_{t_{0}}\left(O, r_{0}\right)} P\left(x, t_{0}\right)}{\sup _{B_{t}\left(O, r_{0}\right) \times\left(0, t_{0}\right]} P(x, t)}=0
$$

where $P(x, t) \doteq \sqrt{\left|R_{\overline{2} 112}\right|^{2}+\left|R_{\overline{2} \overline{1} 12}\right|^{2}}$.

Proof of Theorem 4.5. The proof is motivated by a result of Chen (Theorem 3.1 on p. $371[10]$ ), where an important interior estimate for the Ricci flow was proved. See also Simon [35] for related works.

First, note that $R_{\overline{2} \overline{1} 12} \geq 0$ since $\left(\mathbb{C}^{2}, g(t)\right)$ has non-negative complex sectional curvature. If $R_{\overline{2} \overline{1} 12}=0$ at one point $(x, t)$, then by the strong maximum principle it vanishes everywhere, which further implies $R_{\overline{2} 112}=0$ from the evolution equation of $R_{\overline{2} \overline{1} 12}$. Now assume that $P(x, t)>0$ everywhere and there exists a constant $\epsilon_{0}>0$ such that

$$
\liminf _{r_{0} \rightarrow+\infty, t_{0} \rightarrow 0} \frac{\inf _{B_{t_{0}}\left(O, r_{0}\right)} P\left(x, t_{0}\right)}{\sup _{B_{t}\left(O, r_{0}\right) \times\left(0, t_{0}\right]} P(x, t)} \geq \epsilon_{0} .
$$

We will show that there exists a constant $C$ independent of $r_{0}$ such that

$$
P(x, t) \leq \frac{C}{r_{0}^{2}}
$$

whenever $0<t \leq T\left(n, v_{0}\right)$ and $d_{t}(x, O) \leq \frac{r_{0}}{2}$.

Assume that (4.15) is not true. Then there will exist a sequence $\left\{r_{n}\right\}$ tending to infinity and a sequence of points $\left(x_{n}, t_{n}\right)$ with $P\left(x_{n}, t_{n}\right) \geq \frac{4^{n}}{r_{n}^{2}}$ where $t_{n} \rightarrow 0$ and $d_{t_{n}}\left(x_{n}, O\right) \leq \frac{r_{n}}{2}$.

It follows from a point-picking technique of Perelman [29] (see also p. 372 of [10]) that for any fixed $B>0$, picking $n$ large enough if necessary, we can choose $\left(\bar{x}_{n}, \bar{t}_{n}\right)$ such that $0<\bar{t}_{n} \leq t_{n}, d_{\bar{t}_{n}}\left(\bar{x}_{n}, O\right) \leq \frac{3}{4} r_{n}$, and 
$\bar{Q}_{n} \doteq R\left(\bar{x}_{n}, \bar{t}_{n}\right) \geq \frac{4^{n}}{r_{n}^{2}}$, Moreover

$$
0 \leq R(x, t) \leq 4 \bar{Q}_{n}
$$

wherever $0<\bar{t}_{n} \leq t_{n}$ and $d_{t}(x, O) \leq d_{\bar{t}_{n}}\left(\bar{x}_{n}, O\right)+B \bar{Q}_{n}^{-\frac{1}{2}}$.

As in p. 372 of [10], construct a smooth function $\psi: \mathbb{R} \rightarrow[0,1]$ such that $\psi=1$ on $\left(-\infty, d_{\bar{t}_{n}}\left(\bar{x}_{n}, O\right)+\frac{B}{2} \bar{Q}_{n}^{-\frac{1}{2}}\right]$ and $\psi=0$ on $\left[d_{\bar{t}_{n}}\left(\bar{x}_{n}, O\right)+B \bar{Q}_{n}^{-\frac{1}{2}},+\infty\right)$, Moreover, one can assume that $\left|\psi^{\prime}\right| \leq 4 \frac{\bar{Q}_{n}^{\frac{1}{2}}}{B}$ and $\left|\psi^{\prime \prime}\right|+\frac{\left|\psi^{\prime}\right|^{2}}{\psi} \leq 32 \frac{\bar{Q}_{n}}{B^{2}}$.

Denote by $u(x, t)=\psi\left(d_{t}(x, O)\right)\left(\left|R_{\overline{2} 112}\right|^{2}+\left|R_{\overline{2} \overline{1} 12}\right|^{2}\right)$. We have

$$
\begin{aligned}
\left(\frac{\partial}{\partial t}-\Delta\right) u= & \left\{\psi^{\prime}\left[\left(\frac{\partial}{\partial t}-\Delta\right) d_{t}\right]+\left(\frac{|\psi|^{2}}{\psi}-\psi^{\prime \prime}\right)\right\}\left(\left|R_{\overline{2} 112}\right|^{2}+\left|R_{\overline{2} \overline{1} 12}\right|^{2}\right) \\
& +2 \psi\left[| R _ { \overline { 2 } 1 1 2 } | ^ { 2 } \left(2 R_{\overline{2} \overline{1} 12}+R_{1 \overline{1} 2 \overline{2}}-R_{1 \overline{1} 2 \overline{2}}+R_{2 \overline{2} 2 \overline{2})]}\right.\right. \\
& +2 \psi\left[R_{\overline{2} \overline{1} 12}{ }^{3}+R_{\overline{2} \overline{1} 12}\left|R_{\overline{2} 112}\right|^{2}\right. \\
& +R_{\overline{2} \overline{1} 12}^{2}\left(2 R_{1 \overline{1} 2 \overline{2}}+R_{1 \overline{1} 2 \overline{2}}+R_{2 \overline{2} 2 \overline{2})]}\right. \\
& -2 \psi\left(\left|\nabla R_{\overline{2} \overline{1} 12}\right|^{2}+\left|\nabla R_{\overline{2} \overline{1} 12}\right|^{2}\right),
\end{aligned}
$$

$$
\frac{d^{+} u_{\max }(t)}{d t} \leq\left(16 \frac{\bar{Q}_{n}}{B}+32 \frac{\bar{Q}_{n}}{B^{2}}\right) P(x, t)+32 u_{\max }(t) \bar{Q}_{n}
$$

In the above we used, by (4.16), that $\left(\frac{\partial}{\partial t}-\Delta\right) d_{t} \geq-4 \bar{Q}_{n}^{\frac{1}{2}}$ when $d_{t}(x, O) \geq$ $\bar{Q}_{n}^{\frac{1}{2}}$, from Lemma 8.3 on p. 20 of [29].

It follows from (4.18) that:

$$
\frac{d^{+}}{d t}\left(\mathrm{e}^{-32 \bar{Q}_{n} t} u_{\max }(t)\right) \leq 64 \frac{\bar{Q}_{n}}{B} \mathrm{e}^{-32 \bar{Q}_{n} t} \sup _{B_{t}\left(O, r_{0}\right) \times\left(0, \bar{t}_{n}\right]} P(x, t),
$$

where we use $d(x, t) \leq d_{\bar{t}_{n}}\left(\bar{x}_{n}, O\right)+B \bar{Q}_{n}^{-\frac{1}{2}} \leq r_{0}$ for $n$ large.

Note that Theorem 4.1 guarantees that $\bar{t} \bar{Q} \leq c\left(2, v_{0}\right)$, so (4.19) implies that

$$
u_{\max }(\bar{t}) \leq 2 \frac{\mathrm{e}^{32 c\left(2, v_{0}\right)}-1}{B} \sup _{B_{t}\left(O, r_{0}\right) \times\left(0, \bar{t}_{n}\right]} P(x, t) .
$$

However, since $B$ can be chosen to be arbitrarily large, we get a contradiction with the assumption (4.14). Such a contradiction implies that (4.15) must be true. However (4.15) again contradicts to the assumption 
$P(x, t)>0$ after taking $r_{0}$ approaching to the infinity. To sum up, we have completed the proof of Theorem 4.5.

Note that that the constant $c\left(n, v_{0}\right)$ in Theorem 4.1 will be 0 if $v_{0}$ is the volume of a standard unit ball in Euclidean space. Indeed, starting from a standard flat metric on $\mathbb{R}^{n}$, it is easy to see that (from Theorem 3.1 of [10] for example) that Cabezas-Rivas and Wilking's Ricci flow is a flat one. Intuitively it is reasonable to expect that $c\left(n, v_{0}\right)$ is close to zero if $v_{0}$ is close to the volume of a unit Euclidean ball.

Assume that $\left(\mathbb{C}^{2}, g(t)\right)$ is a Kähler-Ricci flow with $U(n)$-symmetry. Then we certainly have $R_{\overline{2} 112}=R_{\overline{2} \overline{1} 12}=0$. In addition, by Lemma 3.7, we know that the term $\int_{0}^{r} \operatorname{Ric}\left(\frac{\partial}{\partial s}, \frac{\partial}{\partial s}, t\right) d s$ is bounded in the spacetime. The following proposition shows that conversely the boundedness of $R_{\overline{2} 112}, R_{\overline{2} \overline{1} 12}$, and $\int_{0}^{r} \operatorname{Ric}\left(\frac{\partial}{\partial s}, \frac{\partial}{\partial s}, t\right) d s$ in spacetime will be sufficient to ensure the existence of a Kähler-Ricci flow, provided that $c\left(2, v_{0}\right)$ is suitably small.

Proposition 4.6. Under the same assumption as in Theorem 4.5, assume that the constant (4.1) satisfies $c\left(2, v_{0}\right) \leq \frac{1}{32}$, and there exists a constant $t_{1}$ such that $R_{\overline{2} \overline{1} 12}$ and $R_{\overline{2} 112}$ are bounded on $\mathbb{C}^{2} \times\left(0, t_{1}\right]$. In addition, assume that there exists a constant $C_{1}$ which depends on $g(t)$ for $t \in\left(0, t_{1}\right]$ such that

$$
\int_{0}^{r} \operatorname{Ric}\left(\frac{\partial}{\partial s}, \frac{\partial}{\partial s}, t\right) d s \leq C_{1}
$$

holds for all $r>0$ and $t \in\left(0, t_{1}\right]$. Then the Ricci flow $\left(\mathbb{C}^{2}, g(t)\right)$ constructed by Cabezas-Rivas et al. is a $U(n)$-invariant Kähler-Ricci flow as long as it exists.

Proof of Proposition 4.6. Under the assumption (4.21), tracing the proof of Lemma 8.3 on p. 20 of [29], we will get that, for any $t \in\left(0, t_{1}\right]$, the distance function $d_{t}(x, O)$ satisfies the following estimates:

$$
\left(\frac{\partial}{\partial t}-\Delta_{t}\right) d_{t}(x, O) \geq-4\left(C_{1}+\frac{1}{r_{0}}\right)
$$

whenever $d_{t}(x, O)>r_{0}$.

Define $v(x, t)=\eta P(x, t)=\eta\left(\frac{d_{t}(x, O)}{r_{0}}\right)\left(\left|R_{\overline{2} 112}\right|^{2}+\left|R_{\overline{2} \overline{1} 12}\right|^{2}\right)$, where $\eta$ is a cut-off function with $\eta=1$ on $(-\infty, 1], \eta=0$ on $[2,+\infty)$, and $\left|\eta^{\prime}\right|+\left|\eta^{\prime \prime}\right|+$ $\frac{\left|\eta^{\prime}\right|^{2}}{\eta} \leq 16$. 
A similar computation shows that there exists a constant $C_{2}>0$ such that

$$
\frac{d^{+} v_{\max }(t)}{d t} \leq 32 \frac{c\left(2, v_{0}\right)}{t} v_{\max }(t)+\left(\frac{32}{r_{0}^{2}}+\frac{4 C_{1}}{r_{0}}\right) C_{2},
$$

where we use the boundedness of $P(x, t)$.

Note that we have assumed that $32 c\left(2, v_{0}\right)<1$. Integrating (4.23) on $\left[0, t_{1}\right]$, we will arrive at:

$$
v_{\max }\left(t_{1}\right) \leq \frac{t_{1}}{1-32 c\left(2, v_{0}\right)}\left(\frac{32}{r_{0}^{2}}+\frac{4 C_{1}}{r_{0}}\right) C_{2} .
$$

By taking $r_{0} \rightarrow+\infty$, we get $P(x, t)=0$ everywhere. Now the Proposition 4.6 follows from Lemma 4.2.

Remark 4.7. We believe that a more refined analysis on curvature evolution on the double covers will imply that either (4.21) is automatically true or $(4.13)$ contradicts to $P(x, t)>0$ on the limit flow $\left(\mathbb{C}^{2}, g(t)\right)$. We will study the existence of complete Kähler-Ricci flow in a more general context in future works.

\section{Acknowledgements}

We would like to thank Bennett Chow and Lei Ni for their encouragement. Bo Yang is grateful to Guoyi Xu for helpful discussions on [10].

\section{References}

[1] S. Bando, On the classification of three-dimensional compact Kaehler manifolds of nonnegative bisectional curvature, J. Diff. Geom. 19(2) (1984), 283-297.

[2] S. Brendle and R. Schoen, Manifolds with 1/4-pinched curvature are space forms, J. Amer. Math. Soc. 22(1) (2009), 287-307.

[3] E. Cabezas-Rivas and B. Wilking, How to produce a Ricci Flow via Cheeger-Gromoll exhaustion, Arxiv:1107.0606.

[4] H.-D. Cao, Existence of gradient Kähler-Ricci solitons, Elliptic and parabolic methods in geometry (Minneapolis, MN, 1994), 1-16, A K Peters, Wellesley, MA, 1996.

[5] H.-D. Cao, Limits of solutions to the Kähler-Ricci flow, J. Diff. Geom. 65 (1997), 257-272. 
[6] A. Chau and L.-F. Tam, Gradient Kähler-Ricci solitons and complex dynamical systems, Recent progress on some problems in several complex variables and partial differential equations 43-52, Contemp. Math., 400, Amer. Math. Soc., Providence, RI, 2006.

[7] A. Chau and L.-F. Tam, On the complex structure of Kähler manifolds with nonnegative curvature, J. Diff. Geom. 73(3) (2006), 491-530.

[8] A. Chau and L.-F. Tam, Non-negatively curved Kähler manifolds with average quadratic curvature decay, Comm. Anal. Geom. 15(1) (2007), $121-146$.

[9] A. Chau and L.-F. Tam, On the Steinness of a class of Kähler manifolds J. Diff. Geom. 79(2) (2008), 167-183.

[10] B.-L. Chen, Strong uniqueness of the Ricci flow, J. Diff. Geom. 82(2) (2009), 363-382.

[11] B.-L. Chen, S.-H. Tang and X.-P. Zhu, A uniformization theorem for complete non-compact Kähler surfaces with positive bisectional curvature, J. Diff. Geom. 67(3) (2004), 519-570.

[12] B.-L. Chen and Zhu, X-P. On complete noncompact Kähler manifolds with positive bisectional curvature, Math. Ann. 327(1) (2003), 1-23.

[13] B. Chow, S.-C. Chu, D. Glickenstein, C. Guenther, J. Isenberg, T. Ivey, D. Knopf, P. Lu, F. Luo and L. Ni, The Ricci flow: techniques and applications. Part II. Analytic aspects, Mathematical Surveys and Monographs, 144, American Mathematical Society, Providence, RI, 2008. $\mathrm{xxvi}+458 \mathrm{pp}$.

[14] X.-Q. Fan, A uniqueness result of Kähler Ricci flow with an application, Proc. Amer. Math. Soc. 135(1) (2007), 289-298.

[15] G. Giesen and P. Topping, Existence of Ricci flows of incomplete surfaces, Comm. Partial Diff. Equ. 36 (2011), 1860-1880.

[16] R.E. Greene and H. Wu, Analysis on noncompact Kähler manifolds. Several complex variables (Proc. Sympos. Pure Math., XXX, Part 2, Williams Coll., Williamstown, Mass., 1975), 69-100. Amer. Math. Soc., Providence, R.I., 1977.

[17] R.S. Hamilton, Three-manifolds with positive Ricci curvature, J. Diff. Geom. 17(2) (1982), 255-306.

[18] R.S. Hamilton, Four-manifolds with positive curvature operator, J. Diff. Geom. 24(2) (1986), 153-179. 
[19] R.S. Hamilton, The formation of singularities in the Ricci flow, Surveys in differential geometry, II (Cambridge, MA, 1993), 7-136, Int. Press, Cambridge, MA, 1995.

[20] J. Isenberg, R. Mazzeo and N. Sesum, Ricci flow in two dimensions, Surveys in geometric analysis and relativity, Adv. Lect. Math. (ALM), 20, Int. Press, Somerville, MA, 2011, 259-280.

[21] P.F. Klembeck, A complete Kähler metric of positive curvature on $\mathbb{C}^{n}$, Proc. Amer. Math. Soc. 64(2) (1977), 313-316.

[22] N. Mok, An embedding theorem of complete Kähler manifolds of positive bisectional curvature onto affine algebraic varieties, Bull. Soc. Math. France 112(2) (1984), 197-250.

[23] N. Mok, The uniformization theorem for compact Kähler manifolds of nonnegative holomorphic bisectional curvature, J. Diff. Geom. 27(2) (1988), 179-214.

[24] N. Mok, Y-T. Siu and S.-T. Yau, The Poincaré-Lelong equation on complete Kähler manifolds, Composit. Math. 44(1-3) (1981), 183-218.

[25] L. Ni, Ricci flow and nonnegativity of sectional curvature, Math. Res. Lett. 11(5-6) (2004), 883-904.

[26] L. Ni, Y. Shi and L.-F. Tam, Poisson equation, Poincaré-Lelong equation and curvature decay on complete Kähler manifolds, J. Diff. Geom. $\mathbf{5 7}(2)$ (2001), 339-388.

[27] L. Ni and L.-F. Tam, Kähler-Ricci flow and the Poincaré-Lelong equation, Comm. Anal. Geom. 12(1-2) (2004), 111-141.

[28] L. Ni and J. Wolfson, Positive complex sectional curvature, Ricci flow and the differential sphere theorem, arXiv:0706.0332v1.

[29] G. Perelman, The entropy formula for the Ricci flow and its geometric applications, arXiv:math/0211159.

[30] A.M. Petrunin, An upper bound for the curvature integral, (Russian) Algebra i Analiz 20(2) (2008), 134-148 translation in St. Petersburg Math. J. 20(2) (2009), 255-265.

[31] F. Schulze and M. Simon, Expanding solitons with non-negative curvature operator coming out of cones, arXiv:1008.1408. 
[32] W.-X. Shi, Deforming the metric on complete Riemannian manifolds, J. Diff. Geom. 30(1) (1989), 223-301.

[33] W.-X. Shi, Ricci deformation of the metric on complete noncompact Kähler manifolds, PhD thesis, Harvard University, 1990, p. 203

[34] W.-X. Shi, Ricci flow and the uniformization on complete noncompact Kähler manifolds, J. Diff. Geom. 45(1) (1997), 94-220.

[35] M. Simon, Local results for flows whose speed or height is bounded by c/t, Int. Math. Res. Not. IMRN 2008, Art. ID rnn 097, p. 14.

[36] Y.T. Siu, Pseudoconvexity and the problem of Levi, Bull. Amer. Math. Soc. 84(4) (1978), 481-512.

[37] H.-H. Wu and F. Zheng, Examples of positively curved complete Kähler manifolds, Geometry and Analysis Volume I, Advanced Lecture in Mathematics 17, Higher Education Press and International Press, Beijing and Boston, 2010, pp. 517-542.

[38] L.-F. Wu, The Ricci flow on complete $\mathbf{R}^{2}$, Comm. Anal. Geom. 1(3-4) (1993), 439-472.

[39] B. Yang, On a problem of Yau regarding a higher dimensional generalization of the Cohn-Vossen inequality, Math. Ann. 355(2) (2013), 765-781.

[40] S.-T. Yau, Open problems in geometry, Chern-a great geometer of the twentieth century, International Press, Hong Kong, 1992, pp. 275-319.

[41] S.-T. Yau, A review of complex differential geometry, Several complex variables and complex geometry, Part 2 (Santa Cruz, CA, 1989), Proc. Symp. Pure Math., 619-625, Vol 52, Part II, American Mathematical Society, 1991.

[42] T. Yokota, Curvature integrals under the Ricci flow on surfaces, Geom. Dedicata 133 (2008), 169-179.

[43] F. Zheng, First Pontrjagin form, rigidity and strong rigidity of nonpositively curved Kähler surface of general type, Math. Z., 220(2) (1995), 159-169.

Department of MATHEMATICS

University of California, San Diego

LA Jolla, CA 92093, USA

E-mail address: b5yang@math.ucsd.edu 
Center for Mathematical Sciences

ZHEJIANG UNIVERSITY

Hangzhou, Zhejiang, 310027, China

AND

Department of Mathematics

The Ohio State University

231 West 18th Avenue

Columbus, OH 43210, USA

E-mail address: zheng@math.ohio-state.edu

Received May 31, 2012 